\title{
Abhandlung
}

Karina Grömer*, Gert Goldenberg, Johanna Banck-Burgess, Margarita Gleba, Regina Hofmann-de Keijzer, Maarten van Bommel, Ineke Joosten, Matthias Mehofer, Kurt Nicolussi, Ulrike Töchterle

\section{Textilreste aus einem spätbronzezeitlichen Bergbaurevier bei Radfeld in Nordtirol - Sekundärnutzung von Stoffen zur Abdichtung}

https://doi.org/10.1515/pz-2017-0014

\begin{abstract}
Zusammenfassung: Im traditionsreichen Bergbaugebiet von Schwaz/Brixlegg im Nordtiroler Unterinntal fand während der späten Bronzezeit und frühen Eisenzeit ein umfangreicher Bergbau auf Kupfererze statt, der zahlreiche Spuren im Gelände hinterlassen hat. Montanarchäologische Forschungsprojekte der Universität Innsbruck, gefördert vom österreichischen Wissenschaftsfonds FWF und vom Tiroler Wissenschaftsfonds TWF, untersuchen seit den 1990er Jahren das prähistorische Montanwesen in diesem Raum. $\mathrm{Zu}$ den Forschungszielen gehören die Rekonstruktion der metallurgischen Produktionskette vom Erzabbau über die Aufbereitung bis hin zur Erzverhüttung und Gewinnung von Rohkupfer sowie der
\end{abstract}

\footnotetext{
*Corresponding author: Karina Grömer, Naturhistorisches Museum Wien, Prähistorische Abteilung, Österreich.

E-Mail: karina.groemer@nhm-wien.ac.at

Gert Goldenberg, Universität Innsbruck, Institut für Archäologien FZ HiMAT, Österreich. E-Mail: gert.goldenberg@uibk.ac.at

Johanna Banck-Burgess, Landesamt für Denkmalpflege

Baden-Württemberg, Esslingen, Deutschland.

E-Mail: johanna.banck-burgess@rps.bwl.de

Margarita Gleba, University of Cambridge, McDonald Institute

for Archaeological Research, Großbritannien. E-Mail:

mygleba@yahoo.com

Regina Hofmann-de Keijzer, Universität für angewandte Kunst Wien, Abteilung Archäometrie, Österreich.

E-Mail: Regina.Hofmann@uni-ak.ac.at

Maarten van Bommel, Cultural Heritage Agency of the Netherlands, Sector Research Movable Heritage. E-Mail: M.R.vanBommel@uva.nl Ineke Joosten, (RCE Amsterdam), Niederlande. E-Mail:

I.Joosten@cultureelerfgoed.nl

Matthias Mehofer, VIAS - Vienna Institute for Archaeological Science, Österreich. E-Mail: mathias.mehofer@univie.ac.at Kurt Nicolussi, Universität Innsbruck, Institut für Geographie FZ HiMAT, Österreich. E-Mail: kurt.nicolussi@uibk.ac.at Ulrike Töchterle, Universität Innsbruck, Institut für Archäologien FZ HiMAT, Österreich. E-Mail: ulrike.toechterle@uibk.ac.at
}

Arbeits- und Lebenswelt der prähistorischen Berg- und Hüttenleute. Die bislang prospektierten und in Ausschnitten archäologisch untersuchten Befunde belegen einen zeitlichen Schwerpunkt der Bergbauaktivitäten im 12. bis 8. Jh. v. Chr. Auf einem Verhüttungsplatz bei Radfeld (Mauk A) fanden sich 1997 bei der Freilegung einer „Waschrinne“ zur nassmechanischen Aufbereitung von Schlacken einige gut erhaltene Textilfragmente. Der archäologische Befund sowie die Textilfunde, Analysen zu Textiltechnik, Fasermaterial und Farbstoffen werden im folgenden Beitrag vorgestellt. Zudem werden die funktionelle Interpretation und kulturgeschichtliche Einordnung der Textilfunde diskutiert.

Schlüsselworte: Radfeld; Nordtirol; Spätbronzezeit; Kupfergewinnung; Textilien; Funktion

Résumé: Dans la célèbre région minière de Schwaz/ Brixlegg en Tyrol (vallée de l'Inn) se trouvent de nombreuses traces de travaux miniers datant de l'Âge du Bronze final à l'Âge de Fer ancien. L'exploitation de l'époque se concentrait sur le cuivre gris, matière première locale pour la production du cuivre. Depuis 1993 des projets de recherches interdisciplinaires sont effectués à l'Université de Innsbruck sous la promotion du Wissenschaftsfonds autrichien (FWF) et tyrolien (TWF). Les objectifs de ces recherches comprennent la reconstruction de la chaîne opératoire pour la production du cuivre (extraction du minerai, traitement et métallurgie) aussi bien que du monde du travail et du cadre de vie des communautés de mineurs préhistoriques. La datation de ces activités tombe dans la période du $12^{\text {2ème }}$ au $8^{\text {ème }}$ siècle av. J.-C. Une fouille archéologique sur un site de métallurgie extractive (Radfeld, Mauk A, 1997) a mis au jour les vestiges d'une installation de lavage où des scories de cuivre finement broyées ont été lavées pour récupérer des inclusions riches en cuivre. Dans ce contexte, quelques fragments de textile bien conservé ont pu être récupérés. Le 
textile, le matériau en lui-même, ses colorants et la technologie de sa production sont présentés et discutés dans cette contribution ainsi que l'interprétation fonctionnelle et le contexte (pré-)historique et culturel.

Mots-clés: Radfeld; Tyrol du Nord; l'Âge du Bronze final; metallurgie extractive du cuivre; textiles; fonction

\begin{abstract}
In the traditional mining district Schwaz-Brixlegg in North-Tyrol a large amount of archaeological remains from an extensive Late Bronze Age and Early Iron Age copper ore mining is still visible in the landscape. In 1993 the University of Innsbruck started with mining archaeological investigations in this area in the frame of several research projects, supported by the Austrian Science Fund FWF and the Tyrolian Science Fund TWF. The aim of the research program is to identify and to date prehistoric mining activities and to reconstruct the metallurgical production chain including mining, beneficiation and smelting of copper ores. Also the working and living environment of the prehistoric miners and smelters is studied. So far evidence for mining activities from the $12^{\text {th }}$ to the $8^{\text {th }}$ century BC could be provided. In the frame of archaeological excavations in 1997 at the Late Bronze Age smelting site Mauk A near Radfeld a few well preserved textile fragments could be recovered in the context of a washing installation, where crushed slag had been processed in order to obtain concentrates of copper rich inclusions. In this paper the textile finds will be presented in their archaeological context together with analysis on textile techniques, seam materials and dyestuffs. Furthermore, their functional interpretation and positioning in a larger historical context will be discussed.
\end{abstract}

Keywords: Radfeld; North Tyrol; Late Bronze Age; extractive copper metallurgy; textiles; function

\section{Das Bergbaurevier Mauken bei Radfeld/Brixlegg - der kultur- geschichtliche Kontext}

Das Bergbaurevier Mauken im Unterinntal (Gemeinden Radfeld und Brixlegg) bildet den östlichen Ausläufer der traditionsreichen Bergbauregion Schwaz in Tirol und war im 16. Jh. n. Chr. dem Berggericht Rattenberg zugeordnet $^{1}$. Aus den hier verbreitet auftretenden Kupfererzen (im Wesentlichen antimon- und arsenhaltige Fahlerze der

1 Mutschlechner 1984.
Reihe Tetraedrit/Tennantit) wurde in vorgeschichtlicher Zeit Kupfer, seit dem späten Mittelalter Silber und Kupfer gewonnen. Im Teilrevier Mauken sind die Erzvorkommen von vergleichsweise geringer Größenordnung, so dass in jüngerer Zeit nur kleinere Bergbaubetriebe unterhalten wurden, die sich in der historischen Überlieferung kaum oder gar nicht niedergeschlagen haben. Nicht zuletzt diesem Umstand ist es zu verdanken, dass Spuren des prähistorischen Bergbaus wenig überprägt und stellenweise noch gut erhalten sind und damit eine ideale Ausgangslage für montanarchäologische Forschungen gegeben ist.

Bislang konnten im Bergbaurevier Mauken zahlreiche Abbauspuren über und unter Tage, Aufbereitungsplätze und ein Verhüttungsplatz (,Schmelzplatz“) archäologisch untersucht werden ${ }^{2}$. In der Zusammenschau aller bisher dokumentierten Befunde ist es möglich, die gesamte Produktionskette der Kupfergewinnung vom Erzabbau über die Erzaufbereitung bis hin zur pyrometallurgischen Gewinnung von Kupfer durch Verhüttung der Erze in allen wesentlichen Schritten zu erfassen ${ }^{3}$. Neben der Rekonstruktion der technischen Abläufe lassen sich Aussagen über die Infrastruktur im Montanrevier sowie anhand des Fundmaterials auch wertvolle Informationen zu den Arbeits- und Lebensbedingungen der frühen Bergleutegesellschaft und deren Wirtschaftsweise in der späten Bronzezeit und frühen Eisenzeit gewinnen ${ }^{4}$.

\section{Der spätbronzezeitliche Verhüttungsplatz Mauk A - der archäologische Befund}

1994 wurde bei einer montanarchäologischen Prospektion ein prähistorischer Verhüttungsplatz (Mauk A) im Maukental bei Radfeld entdeckt (Abb. 1). Dieser liegt auf einer kleinräumigen Verebnungsfläche in sonst steilem Gelände und wird von einem kleinen Quellbach durchflossen. Ein hier im Zuge des Forstwegebaus angelegter Drainagegraben ermöglichte die Beobachtung einer vom Waldboden überdeckten Schlackenhalde. Im Profil des Baggergrabens konnten bei der ersten Begehung Sedimentschichten aus Schlackensandablagerungen festgestellt sowie prähistorische Keramikfragmente (Gebrauchskeramik und Teile von Gebläsetöpfen/Verhüttungsdüsen ${ }^{5}$ ) sowie Tierknochen

\footnotetext{
2 Goldenberg/Rieser 2004; Goldenberg 2008; Goldenberg et al. 2012. 3 Goldenberg 2013; 2015.

4 Schibler et al. 2011.

5 Töchterle et al. 2013.
} 


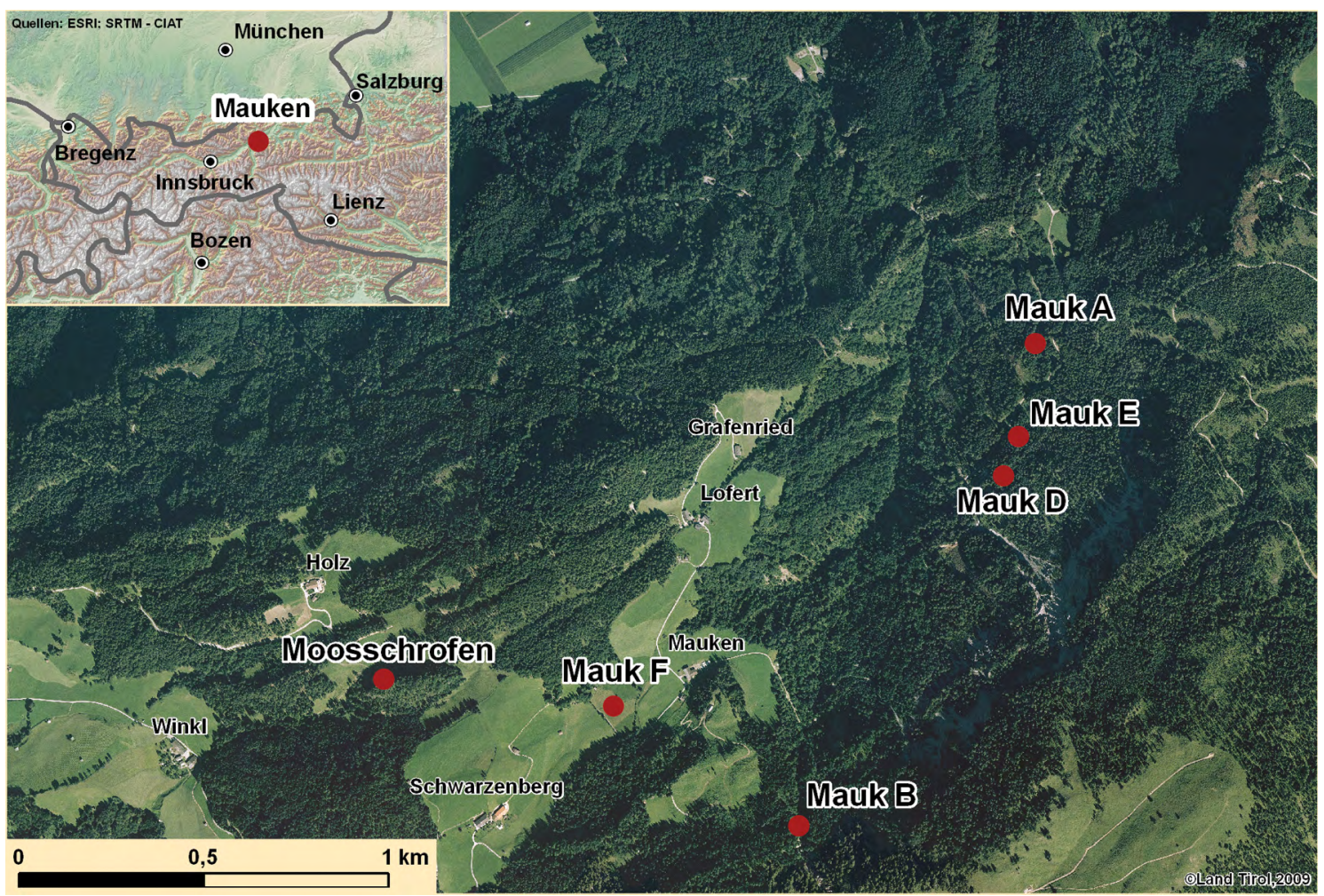

Abb. 1: Das prähistorische Bergbaurevier im Maukental bei Radfeld/Brixlegg mit archäologischen Fund- bzw. Grabungsplätzen (Karte: G. Hiebel/K. Hanke-Univ. Innsbruck; Orthophoto Land Tirol; ESRI; SRTM-CIAT)

(Speiseabfälle) aufgefunden werden. In der Folge fanden mehrere Grabungskampagnen statt, die jeweils vom österreichischen Wissenschaftsfonds FWF im Rahmen größerer Forschungsprojekte gefördert wurden (zuletzt SFB HiMAT, 2007 bis 2012). In der Nähe des Verhüttungsplatzes finden sich zahlreiche feuergesetzte Gruben (Mauk E) und kleinere Pingenfelder (Mauk D), von denen spätbronzezeitliche bis früheisenzeitliche Datierungen vorliegen.

\subsection{Die pyrometallurgischen Anlagen - Röstbetten und Schmelzöfen}

Im Vorfeld der archäologischen Ausgrabungen wurde versucht, mit Hilfe von Magnetfeldmessungen ehemalige pyrometallurgische Anlagen (Schmelzöfen, Röstbetten) im Untergrund $\mathrm{zu}$ lokalisieren ${ }^{6}$. Dies war zunächst nur bedingt erfolgreich, da die gemessenen magnetischen Anomalien vor allem von Schlackenablagerungen verursacht wurden (Abb. 2). Erst im Sommer 2008 konnten schließlich auch die Überreste von zwei Schmelzöfen sowie ein mehrphasig genutztes Röstbett freigelegt und

6 Goldenberg/Rieser 2004; Goldenberg et al. 2012. dokumentiert werden ${ }^{7}$. Damit reiht sich der Befund in eine große Reihe von vergleichbaren montanarchäologischen Fundplätzen in anderen Regionen der Ostalpen $e^{2}{ }^{8}$. Die mineralogische Analyse von Schlacken und weiteren metallurgischen Prozessrückständen belegt die Verarbeitung von Fahlerz-dominierten Kupfererzen ${ }^{9}$, wie sie in der unmittelbaren Umgebung des Verhüttungsplatzes im sogenannten „Schwazer Dolomit“ vorkommen und bergmännisch abgebaut wurden.

\subsection{Die Schlackenhalde}

Die bei der geomagnetischen Vermessung des Verhüttungsplatzes festgestellten Anomalien zeigen in erster Linie die Lage und Ausdehnung der Schlackenhalde an. Diese Interpretation wird durch das Ergebnis von Bohrstockuntersuchungen bestätigt, bei denen ausgedehnte Schlackensandablagerungen in einer Mächtigkeit von bis zu $130 \mathrm{~cm}$ ermittelt wurden. Die zu Sand (Grus) zerkleinerte Kupferschlacke sowie zahlreiche Funde von Reib-

7 Hye et al. 2009; Goldenberg et al. 2012.

8 Weisgerber/Goldenberg 2004.

9 Krismer et al. 2015. 


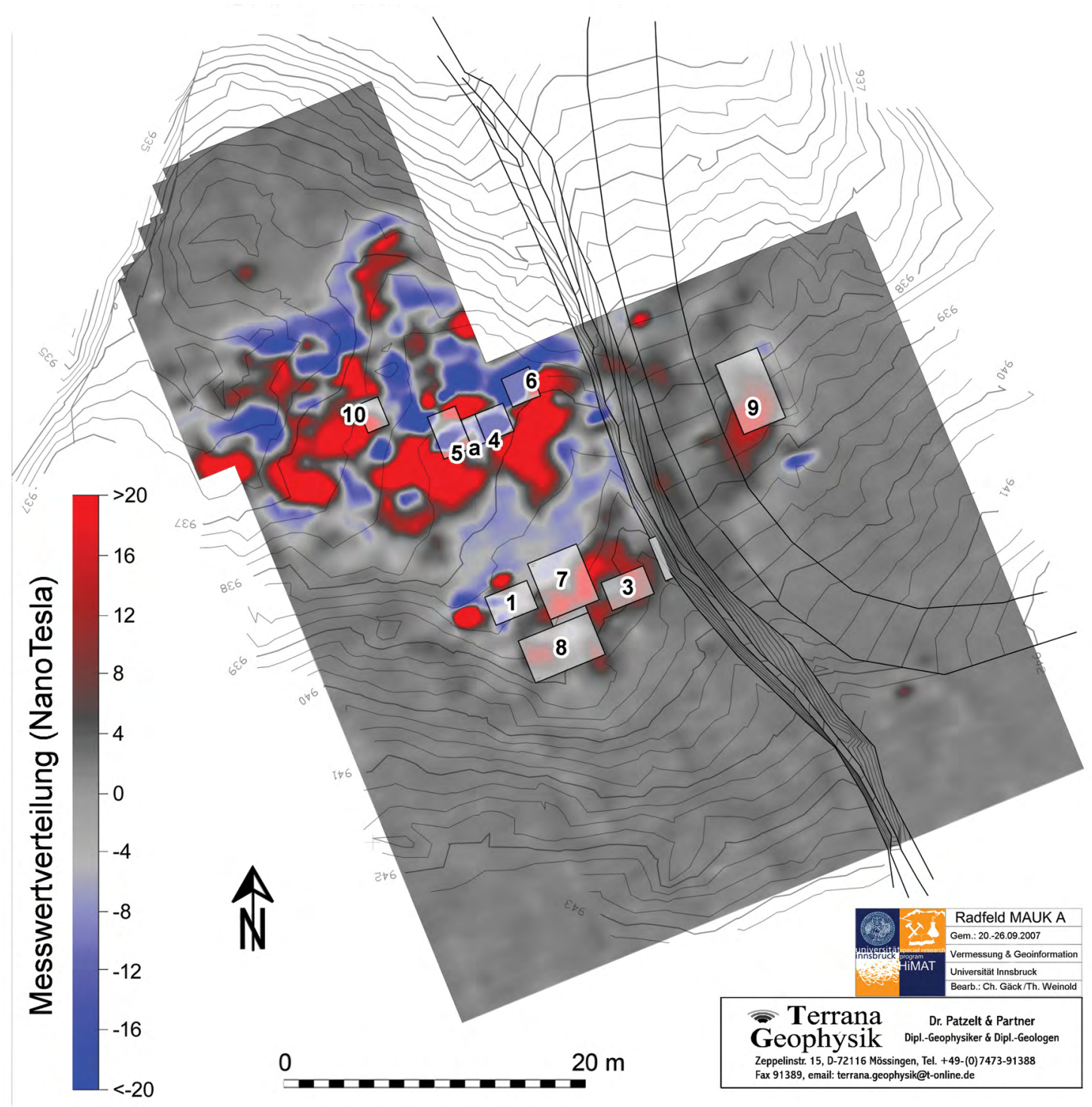

Abb. 2: Geomagnetische Anomalien auf dem Verhüttungsplatz Mauk A mit Lage der Grabungsschnitte (Grafik: Ch. Gäck, Th. Weinold, S. Hye-Univ. Innsbruck; A. Patzelt-Terrana Geophysik-Mössingen)

steinen/Mahlsteinen zeugen von einer systematischen Aufbereitung der beim Verhüttungsprozess erzeugten Schlacken. Der dabei produzierte Schlackensand wurde anschließend über Waschrinnen geleitet, um nach dem Prinzip der Schweretrennung an Kupfer angereicherte Konzentrate zu gewinnen und mit deren Weiterverarbeitung die Ausbeute an Metall zu optimieren. Während die schweren, kupferhaltigen Bestandteile der Schlacken am Boden des Gerinnes zurückblieben, wurde der Rest der leichteren Schlacken weggespült und lagerte sich unterhalb der Waschanlage ab. 1995 und 1997 konnte bei Sondierungsgrabungen in der Schlackensandhalde ein gut erhaltener Befund einer mit Holz verschalten Waschrinne freigelegt und dokumentiert werden (Abb. 3; 4).
Eine Besonderheit des Schlackensandes ist seine konservierende Eigenschaft für organisches Material, hervorgerufen durch die antibakterielle Wirkung der Kupfersalze, die dem Zerfall von organischer Substanz entgegenwirken. So sind z. B. in der Schlackenhalde entsorgte Tierknochen (Speiseabfälle) besonders gut erhalten und durch Kupfersalze meist intensiv grün gefärbt ${ }^{10}$. Für die Archäobotanik und Archäozoologie bilden die im Schlackensand erhaltenen Makroreste von Pflanzen und Kleinlebewesen (Insekten $u$.a.) eine wahre Fundgrube für die Rekonstruktion der damaligen lokalen Umwelt ${ }^{11}$. Bei den

10 Schibler et al. 2011.

11 Heiss/Oeggl 2005; Schatz et al. 2002. 


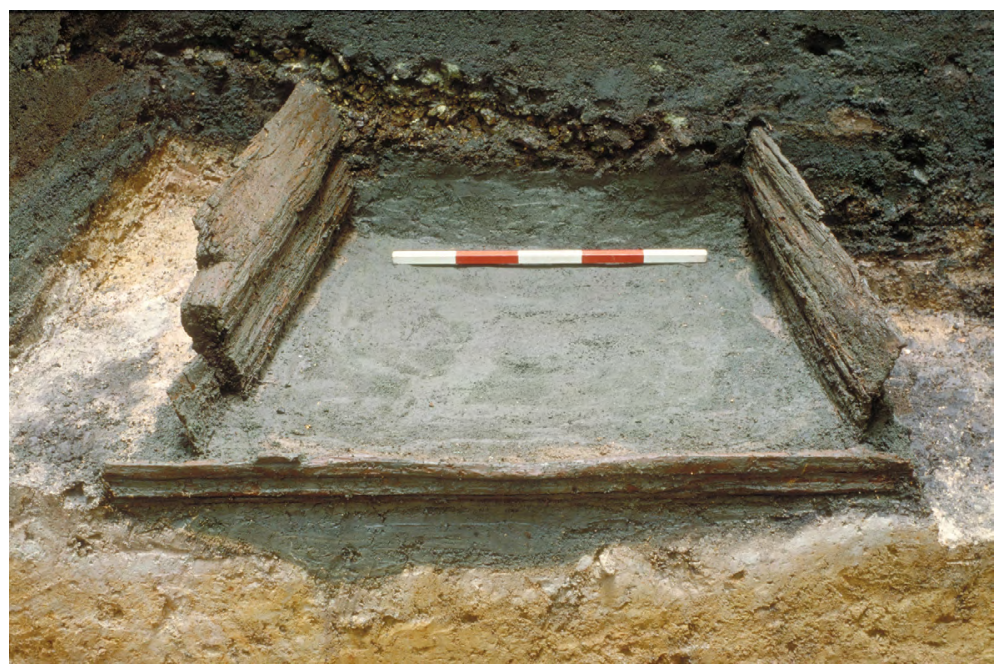

Abb. 3: Unterer Bereich der mit Holzbrettern verschalten Waschrinne, überlagert und konserviert von Schlackensand (Grabung 1997, Foto: G. Goldenberg-Univ. Innsbruck)

Grabungen in der Schlackensandhalde konnten auch gut erhaltene Textilreste aufgefunden werden sowie eine Nähnadel aus Bronze mit Fadenrest (Abb. 11).

\subsection{Die nassmechanische Schlacken- aufbereitung - eine Waschanlage für Schlackensand}

Der komplexe und mehrstufige Prozess der Kupfergewinnung beinhaltet im Falle des Schmelzplatzes Mauk A neben den pyrometallurgischen Verfahren auch eine systematische nassmechanische Aufbereitung von Schlacken. Hierzu wurde von den spätbronzezeitlichen Hüttenleuten im lehmigen Untergrund des Schmelzplatzareals zunächst ein Graben mit leichtem Gefälle angelegt. Der obere Abschnitt des Grabens diente der Zuleitung von Wasser aus dem nahegelegenen Wasserlauf. Der untere Teil des Grabens wurde auf einer Länge von ca. $150 \mathrm{~cm}$ mit Holzbrettern längsseitig eingefasst und am unteren Ende (Auslauf) mit einem quer gestellten Brett abgeschlossen (Abb. 3; 4). Zur Verstärkung der Konstruktion wurde das nördliche Brett mit zwei Rundholzstangen hinterlegt. Der im oberen Abschnitt $30 \mathrm{~cm}$ schmale Einlauf des Gerinnes verbreitert sich im unteren Abschnitt trapezförmig auf eine Breite von $90 \mathrm{~cm}$. Den unteren Abschluss bilden mehrere quer zur Fließrichtung verlaufende Bretter, deren parallele Anordnung und unterschiedliche Höhenlage auf eine mehrfache Ergänzung/Reparatur und damit auf eine mehrphasige Benutzung der Anlage hindeuten. An der nördlichen unteren Ecke des Gerinnes ist eine etwa $10 \mathrm{~cm}$ breite Lücke in der Holzeinfassung festzustellen, die mit einem dünnen Holzbrettchen verschlossen bzw. geöffnet werden konnte. Zweifellos diente dieser im Befund noch erhaltene „Schieber“ zur Regulierung des Wasserstandes bzw. zum Ablassen des Wassers nach Beendigung eines Waschvorgangs (Abb. 4, Markierung 1).

Im vertikalen Profilschnitt durch die Waschanlage wird deutlich, dass diese während ihrer Nutzung sukzessive mit Schlackensand-Sedimenten verfüllt und schließlich außer Betrieb genommen wurde. Im Befund überlagert grobes Pochmaterial von einer Erzaufbereitungstätigkeit die stillgelegte Waschanlage bevor eine weitere Aufsedimentierung bzw. Überdeckung mit Schlackensand erfolgte. Letztere weist auf das ehemalige Vorhandensein weiterer, relativchronologisch jüngerer Waschanlagen hin, die im nahen und höher gelegenen Umfeld der untersuchten Fläche zu erwarten sind.

\subsection{Textilfunde in der Waschanlage}

Eng an die Außenseiten der Holzverschalung angeschmiegt fanden sich während der Freilegung des Befundes an mehreren Stellen Textilfragmente (Abb. 4; Abb. 5), die aufgrund einer starken Verklumpung mit Schlackensand bei der Ausgrabung nicht sofort als solche erkannt werden konnten. Erst nach der Bergung und einer eingehenderen Begutachtung wurden Gewebestrukturen festgestellt. Aufgrund der Befundsituation und des stark fragmentierten, zusammengeballten und teilweise in sich verdrehten Zustands der Fundstücke ist davon auszugehen, dass Teile von ausgedienten Textilien („Lumpen“) eingesetzt wurden um undichte Stellen der Waschanlage 


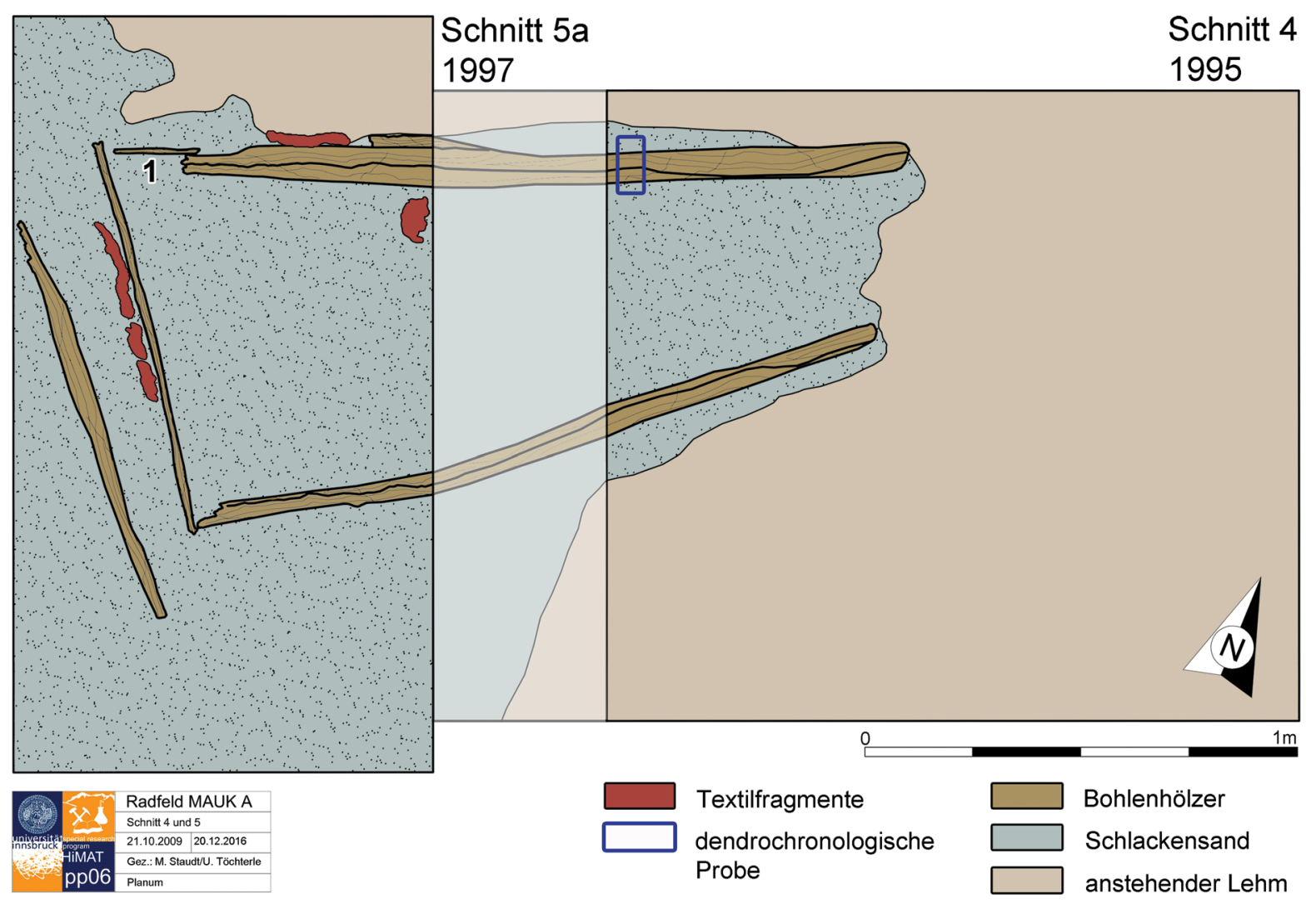

Abb. 4: Plan der freigelegten Waschrinne in Schnitt 4 (1995) und Schnitt 5a (1997) mit Lage der Textilfunde, Schieber zur Regulierung des Wasserabflusses (1) und Entnahmestelle einer Dendro-Probe (Grafik: M. Staudt, U. Töchterle, G. Goldenberg, P. Fliri-Univ. Innsbruck)

abzudichten. Neben dieser naheliegenden Interpretation wäre es prinzipiell auch denkbar, dass Textilbahnen und hier insbesondere Wollstoffe mit flauschigen Fasern am Boden der Waschrinne ausgelegt wurden, um einerseits schwere (kupferreiche) Partikel während des Waschvorgangs zurückzuhalten (vergleichbar mit dem Goldwaschen) und andererseits das so gewonnene kupferhaltige Schwerekonzentrat besser aufnehmen zu können ${ }^{12}$ (Abb. 14). Diese Methode ist seit der Antike bekannt und wird auch von Georgius Agricola in seinen zwölf Büchern de re metallica beschrieben ${ }^{13}$. Ein unmittelbarer Nachweis für die Anwendung dieser Aufbereitungstechnik liegt vom Schmelzplatz Mauk A allerdings nicht vor. Lediglich ein Textilfragment wurde im Innern der Holzkonstruktion vorgefunden.

12 Retournard 2015, 85-86.

13 Agricola 1556.

\subsection{Datierung des Befundes}

Während das Ensemble des Verhüttungsplatzes Mauk A zunächst über ${ }^{14} \mathrm{C}$-Analysen sowie über die Typologie von Kleinfunden grob in das 12./11. Jh. v. Chr. datiert werden kann, liegt mittlerweile auch eine dendrochronologische Datierung einer der 1995 beprobten Holzbohlen aus der Waschanlage vor (Abb. 4; 6). Die Jahrringserie des untersuchten Fichtenholzes ${ }^{14}$ datiert auf der Basis der lokalen Nadelholz-Jahrringchronologie des nahe gelegenen Aufbereitungsplatzes Mauk F ${ }^{15}$ (Schwarzenberg Moos, Abb. 1) 1116-1026 v. Chr. Die Schlägerung kann unter Berücksichtigung des deformierten Randabschnittes mit „um 1010 v. Chr.“ angegeben werden. Mit der präzisen dendrochronologischen Datierung ist es aufgrund des Befundzusammenhangs möglich, auch die Textilfunde zeitlich genauer einzuordnen. Die in der Waschanlage verwendeten Textilfragmente kamen demnach am Ende des 11. Jhs

14 Bohle bbui-1 nach Nicolussi/Pichler 2013; 91 Jahrringe, Jahrringreihe ohne Einbeziehung des gestauchten Holzaußenbereichs der Probe.

15 Nicolussi/Pichler 2013; Nicolussi/Pichler/Thurner 2015. 


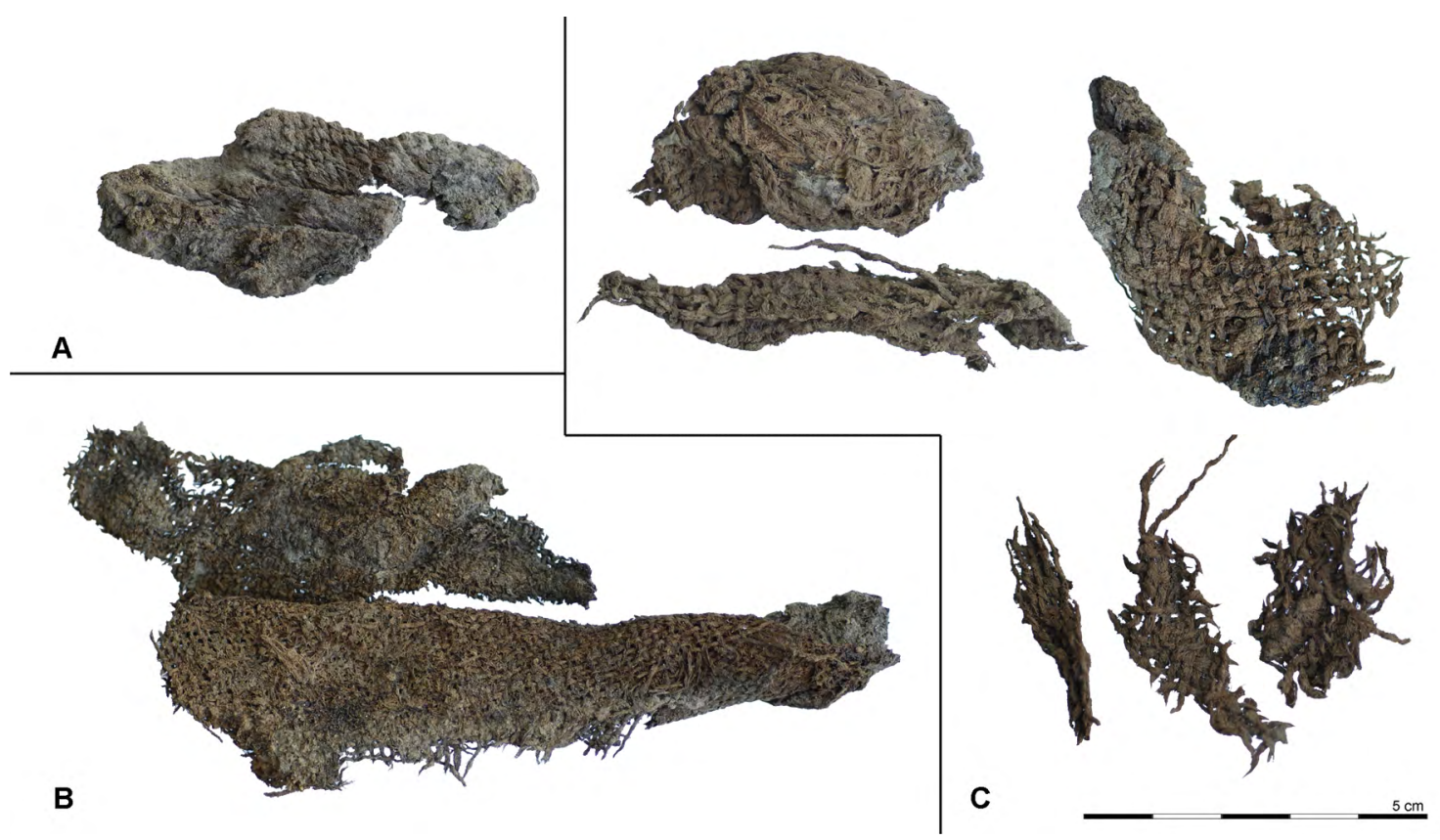

Abb. 5: Textilreste verschiedener Gewebe (A, B, C) nach der Reinigung (Foto G. Goldenberg, U. Töchterle-Univ. Innsbruck)

v. Chr. als Dichtmaterial zum Einsatz. Da es sich aufgrund der Fundsituation und der starken Fragmentierung der Textilreste sehr wahrscheinlich um Abfallstoffe und damit um eine Sekundärnutzung (Recycling) handelt, kann für den primären Gebrauch der Textilien als Bestandteil von Kleidungsstücken die zweite Hälfte des 11. Jhs v. Chr. angenommen werden (1050-1010 v. Chr.).

\section{Die Textilreste}

\subsection{Methodik bei der Konservierung und Analyse der Textilfunde}

Die im Block mit umgebendem Sediment geborgenen Gewebefunde der Grabung 1997 wurden von Johanna Banck-Burgess $^{16}$ (unpubl. Bericht 2000) konservatorisch bearbeitet, freigelegt und analysiert. Die Gewebepartien im Sedimentklumpen waren in ihrer Struktur stellenweise aufgelöst und der Abbau der Fasersubstanz weit fortgeschritten. Durch langsames, kontrolliertes Freispülen mit unterschiedlichen Pipetten konnten schließlich die Fragmente von verschiedenen Geweben dokumentiert werden.

Von den drei vertretenen Geweben wurden jeweils mehrere Proben zur Bestimmung des Materials, der Faserqualität und für Farbstoffanalysen verwendet, die 1998

16 Banck-Burgess 2000. von Penelope Walton Rogers (unpubl. Bericht 1998) durchgeführt wurden. Diese erste Faseranalyse mittels Absorptions-Spektrophotometrie brachte kein Ergebnis, weshalb 2012 abermalig von Regina Hofmann-de Keijzer, Maarten van Bommel und Ineke Joosten mit weiteren Methoden analysiert wurde (unpubl. Bericht 2012). HochleistungsFlüssigkeitschromatographie mit Photo-Dioden-ArrayDetektion (HPLC-PDA) diente dabei zur Farbstoffanalyse, Durchlichtmikroskopie (DLM) und Rasterelektronenmikroskopie mit energie-dispersiver Röntgenanalyse (REMEDX) zur Faser- bzw. Elementanalyse.

Die Faseranalyse an der Bronzenadel erfolgte mittels Rasterelektronenmikroskop, in Zusammenarbeit mit Mathias Mehofer von der VIAS (Vienna Institute for Archaeological Science). Die Fasern der Textilien wurden von verschiedenen Institutionen (unpubl. Berichte BanckBurgess 2000, Walton Rogers 1998, Gleba 2012a, sowie Grömer mit VIAS Wien) analysiert, wobei auf den Nachweis von Wollfasern hin auch Wollfeinheitsmessungen vorgenommen wurden. Die Wollfeinheitsmessungen zur Überprüfung der Faserqualität beruhen auf der Messung der Querschnittsdicke von 100 Fasern pro Faden (Angabe in Mikrometern) mittels Rasterelektronenmikroskop ${ }^{17}$.

Bei der Textilanalyse ${ }^{18}$ wird generell die Bindungsart bestimmt, die Art der Überkreuzung zwischen Schuss-

17 Rast-Eicher 2013, 163-168.

18 Zur Vorgehensweise siehe Walton/Eastwood 1988; auch Grömer 2014, 9-16. 


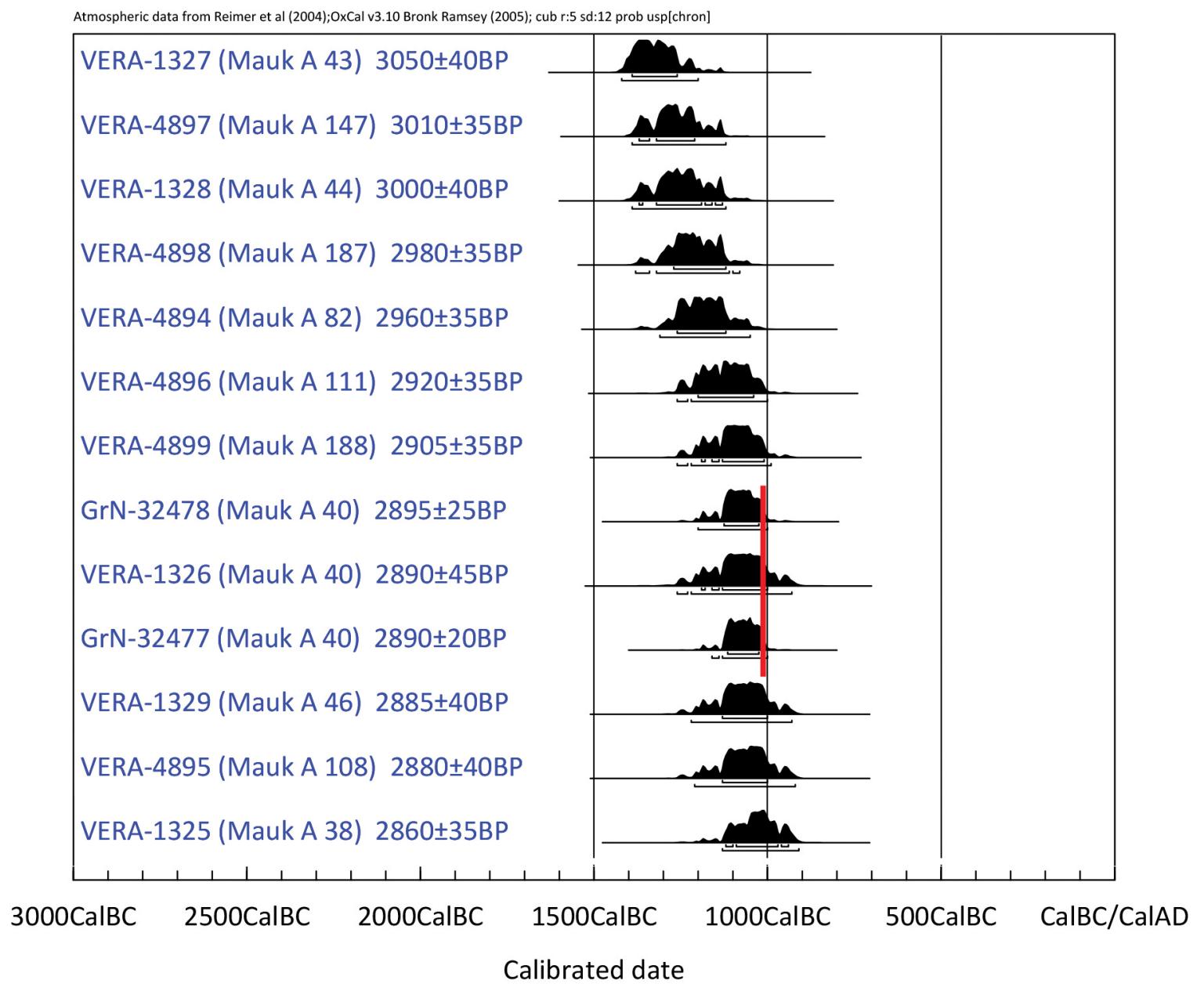

Abb. 6: ${ }^{14} \mathrm{C}$-Daten vom Verhüttungsplatz Mauk $\mathrm{A}$ und dendrochronologische Datierung einer Holzbohle (Grafik: G. Goldenberg, K. Nicolussi-Univ. Innsbruck)

und Kettfäden. Weitere gewebetechnische Details sind die Gewebedichte (Anzahl der Fäden in Kette und Schuss pro $\mathrm{cm})$, die Fadenstärke und die Verdrehungsrichtung des Fadens (wird als s- oder z-Drehung angegeben, nach der Richtung der Schrägstriche in diesen Buchstaben), sowie der Drehwinkel.

Die ersten gewebetechnischen Analysen wurden von Johanna Banck-Burgess im Jahre 2000 an den Geweben noch im feuchten Zustand direkt bei und nach der Konservierung durchgeführt. Eine abermalige Beschreibung erfolgte zehn Jahre später durch das Textil-Team des Projektes CinBA, wobei mit dem Dino-Lite Digital Microscope unter 20- bis 50-facher Vergrößerung sowohl Messungen durchgeführt als auch Mikroskopaufnahmen angefertigt wurden. Hierbei war von Interesse, inwieweit sich Daten wie Fadenstärke oder Gewebedichte durch die Trocknung verändert haben. Dazu sei bemerkt, dass die Unterschiede in den aufgenommenen Maßen (siehe Katalog) durchaus den zu erwartenden Schwankungsbreiten entsprechen.
Es kann also keine signifikante Schrumpfung der Gewebe festgestellt werden. Allgemein ist jedoch anzumerken, dass die Gewebe durch die Trocknung sehr spröde und brüchig geworden sind.

\subsection{Die Gewebefunde - Webtechnik}

Der Fundkomplex besteht aus Fragmenten von mindestens drei unterschiedlichen Geweben (Gewebe A, B und C; Abb. 5 und 7). Die einzelnen Gewebe waren ursprünglich fest in sich zusammengedreht und lagen parallel beieinander. Der Befund und die Reste der drei Gewebe deuten an, dass es sich um einfache Stoffstücke gehandelt hat. Verarbeitungsspuren, wie Schnittkanten, Nähte oder Säume waren nicht erkennbar. In den Gewebefalten von A haben sich zahlreiche holzkohleartige Partikel, Schlackenkörner und Nadeln von Nadelbäumen erhalten. An Fragmenten von Gewebe C fand sich eine teerartige Substanz. 
Gewebe A

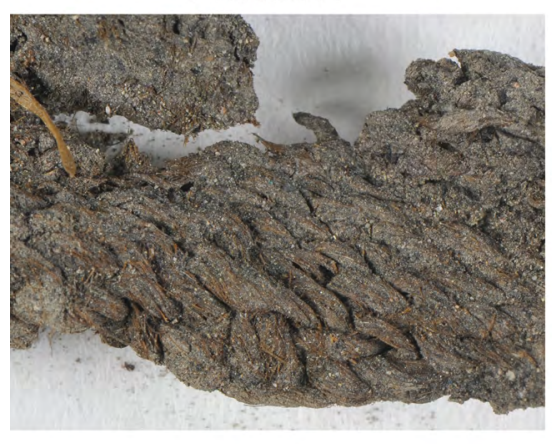

Gewebe B

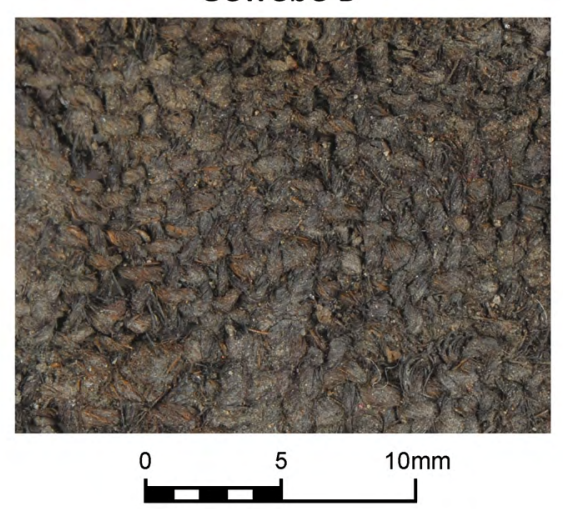

Gewebe C

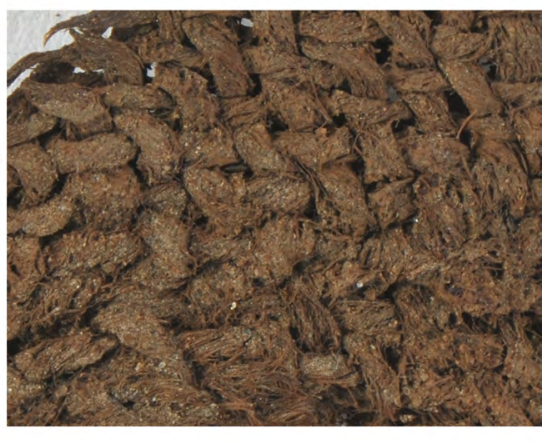

Abb. 7: Radfeld Mauk A, Gewebe A-C. Alle im selben Maßstab (Foto: A. Rausch-NHM Wien)

Alle drei Gewebe sind in Leinwandbindung gefertigt, jedoch sind die herstellungstechnischen Unterschiede zwischen den Geweben in Bezug auf die Gewebequalität auffallend (Abb. 7). Gewebe B hat eindeutig die feinste Qualität mit 0,7-0,9 mm Garnen und einer Webdichte von 10 bis 12 Fäden pro $\mathrm{cm}$. Gewebe $C$ ist hingegen sehr grob mit einer Gewebedichte von 5 Fäden pro $\mathrm{cm}$. Der grobe Eindruck dieses Gewebes wird noch verstärkt durch die sehr ungleichmäßig gesponnenen Fäden, die in der Fadenstärke zwischen 1 und $2 \mathrm{~mm}$ schwanken.

Die Drehrichtung der Garne liegt bei jedem Gewebe in einer anderen Kombination vor. Für Gewebe A wurden in einem Fadensystem z-Garne, im anderen s-Garne verwendet. Für Gewebe B wurden in beiden Fadensystemen ausschließlich z-Garne verwendet, wobei die Fäden teils stark gedreht, teilweise überdreht sind. Gewebe $\mathrm{C}$ wurde ausschließlich mit s-Garnen gefertigt.

Bei Gewebe B sind interessante Details zur Herstellung zu beobachten, im Verlauf eines Fadensystems finden sich verschiedene Webfehler. So sind einmal doppelt eingezogene Garne erkennbar. Zudem springt das Garn einmal über mindestens sechs Eintragsfäden, bindet mit einem ab und springt erneut über mindestens drei Schussfäden. Derartige Webfehler treten beim Weben fast ausschließlich in der Kette auf.

\subsection{Ergebnisse der Faseranalyse}

Die Gewebe vom Verhüttungsplatz Mauk A bei Radfeld bestehen aus Wolle, wobei bei den einzelnen Geweben durchaus Unterschiede festgestellt werden können. Für Gewebe A wurden in Kette und Schuss hellbraune, feine Fasern benützt. Gewebe B besitzt in beiden Fadensystemen Mischfasern (dicke Grannenhaare und feine Unterwolle), wobei das Grundmaterial aus den gleichen Fasern besteht, die auch für Gewebe A verwendet wurden. In einem Fadensystem fanden sich neben diesen Grundfasern zudem hellbraune, gröbere Elemente, im zweiten Fadensystem wurden kräftige, schwarzbraune Fasern entdeckt. Auch Gewebe $C$ besitzt Fasern in Kette und Schuss, die mit Gewebe A und B vergleichbar sind, sowie zusätzlich hellbraune, gröbere Elemente (Abb. 8 rechts). Die anfängliche Vermutung, es könne sich eventuell um Pflanzenfasern handeln, wurde bei einer Untersuchung durch Klaus Oeggl (Universität Innsbruck, Institut für Botanik; in Bericht Banck-Burgess 2000) widerlegt. Gewebe B besaß aufgrund der unterschiedlichen Materialien in Kette und Schuss eine Farbmusterung.

Mittels Wollfeinheitsmessungen können weitere wertvolle Informationen gewonnen werden ${ }^{19}$. Bei dieser Messung werden von 100 Fasern die Durchmesser eruiert und in einem Histogramm dargestellt. Auf diese Weise lassen sich Unterschiede in der Faserqualität sowie verschiedene Haartypen erkennen. Durch Messungen im Rasterelektronenmikroskop ist es möglich, die gröberen Grannenhaare (mit mehr als 100 microns Durchmesser) und die feine Unterwolle (weniger als 60 microns) auseinanderzuhalten (Abb. 8). Mit dieser Methode erarbeitete Michael Ryder ein Schema zur Entwicklung der Schafrassen ${ }^{20}$. Wie bei ihren wilden Vorfahren hatte das Fell der primitiven Schafrassen mehr Grannenhaare als feine Unterwolle. Ryders Hypothese lautet, dass letztere dann jedoch gezielt gezüchtet wurden, um gut verspinnbares Fasermaterial zu erhalten. Die Interpretationsmethodik der Wollfeinheitsmessung wird seitdem verfeinert ${ }^{21}$.

Die Wollfeinheitsanalysen von Penelope Walton Rogers (unpubl. Bericht 1998) detektierten in den Radfeld-

19 Gleba 2012a; 2012b.

20 Ryder 1964; 1983.

21 Siehe Gleba 2012b; Rast-Eicher 2008, 121-136; 2013. 

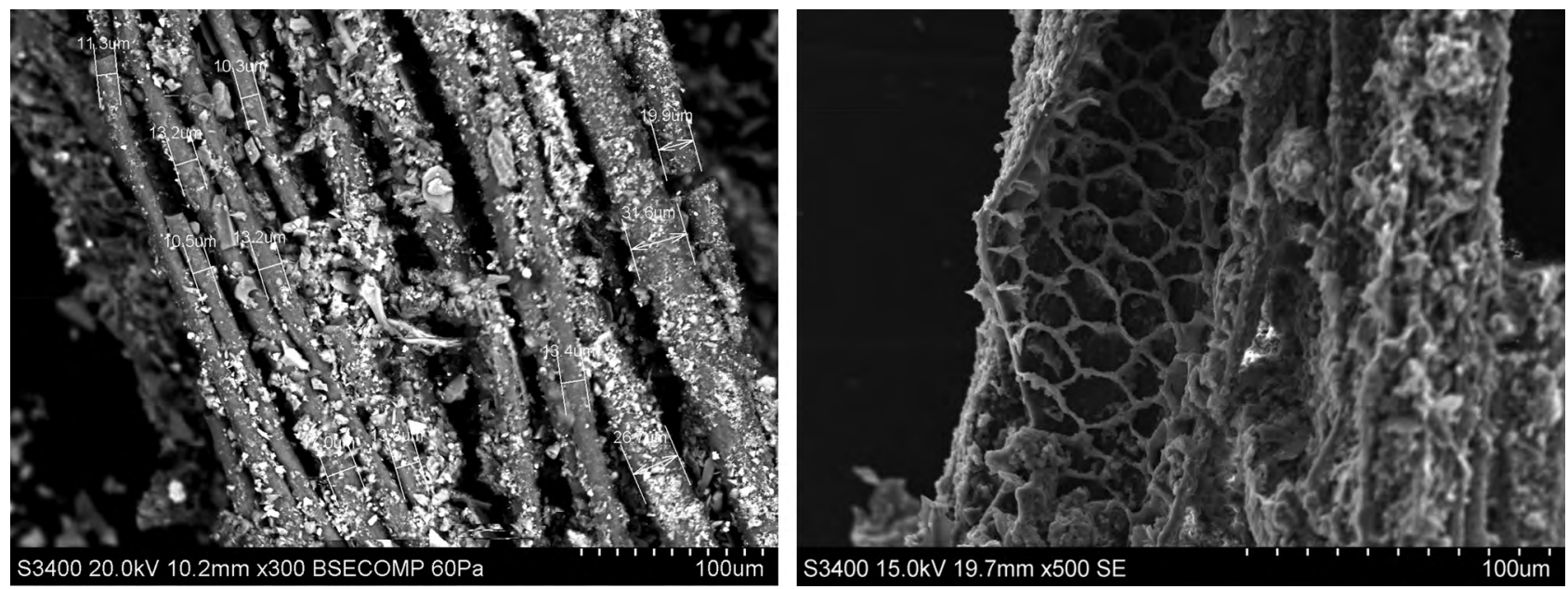

Abb. 8: Messungen an den Fasern von Gewebe A (links). Grannenhaar von Gewebe C mit interner Struktur (rechts) (REM-Fotos: M. Gleba-Univ. Cambridge)

Textilien feine Unterwolle mit 14 bis 20 microns Faserdurchmesser, sowie grobe Grannenhaare mit über 100, teils bis zu 189 microns. Die neuen Wollfeinheitsanalysen von Margarita Gleba ${ }^{22}$ bestätigen im Großen und Ganzen dieses Bild (Abb. 9), vor allem was die feinen Fasern zwischen 9 und 25 microns angeht. Gröbere Grannenhaare wurden vor allem bei Gewebe C identifiziert.

Die Gewebe aus Radfeld Mauk A zeigen allgemein das Bild der typischen gröberen bronzezeitlichen Wollvliese, die dem heutigen Soay-Schaf entsprechen. Das SoaySchaf ${ }^{23}$ hat ebenso Feinwolle mit einem hohen Peak unter 20 microns, jedoch mehr Grannenhaare. Möglicherweise liegt die Abweichung an den bronzezeitlichen Gewinnungs- und Aufbereitungsmethoden, bei denen bewusst die nicht so gut verspinnbaren und steifen, groben Haare ausgesondert wurden ${ }^{24}$.

\subsection{Ergebnisse der Farbstoffanalysen}

Mit HPLC-PDA stand die derzeitige Standardmethode zur Farbstoffanalyse von prähistorischen und historischen Textilien zur Verfügung ${ }^{25}$. Farbige Komponenten können damit schon in sehr geringen Konzentrationen detektiert werden. Es wurden in allen Proben von Radfeld Mauk A gelbe Komponenten entdeckt, sowie rote Komponenten in

22 Gleba 2012a; 2012b.

23 Rast-Eicher 2013, 177.

24 Vgl. Grömer 2016, 65-71.

25 Vgl. Hofmann-de Keijzer 2016, 144-147. den Geweben A und B (Abb. 10) ${ }^{26}$. Für die gelbe Komponente aus Gewebe A ist es nicht klar, ob es sich überhaupt um einen Farbstoff handelt. Die zwei gelben Komponenten aus den Geweben B und C haben ein ähnliches Spektrum wie Maklurin. Auch bei diesen Komponenten ist es nicht ganz sicher, ob es sich um Farbstoffe handelt. Die beiden roten Komponenten aus den Geweben A und B sind vermutlich Farbstoffe, aber aus Mangel an entsprechendem Vergleichsmaterial konnte man sie weder identifizieren noch einer Pflanze zuordnen. Außer den farbigen Verbindungen wurden in allen drei Proben farblose Komponenten nachgewiesen, die nicht im sichtbaren Bereich, sondern nur im UV-Bereich erkennbar sind. Bei den im Jahre 2012 durchgeführten Untersuchungen ${ }^{27}$ wurden mit REM-EDX Spuren von Kupfer festgestellt. Dieses chemische Element dürfte aus der Umgebung in die Textilien gelangt und auf das Lagerungsmilieu zurückzuführen sein (kupferhaltiger Schlackensand).

Falls es sich bei den farbigen Komponenten aus den Geweben von Radfeld Mauk A tatsächlich um Farbstoffe handeln sollte, die zum Färben benutzt worden sind, stammen diese nicht aus allgemein bekannten Färbepflanzen, sondern von bisher nicht identifizierten Pflanzen, die eventuell lokal zum Färben benutzt worden sein könnten. Komponenten können aber auch aus Materialien stammen, welche am Fundort um die Textilien gelagert waren. Die gelben Komponenten könnten unter anderem aus den Moosen stammen, die neben den Textilien gefunden wurden.

26 Eine erste Farbstoffanalyse von Penelope Walton Rogers (1998) ergab, dass alle Proben ungefärbt waren.

27 Hofmann-de Keijzer et al. 2012. 

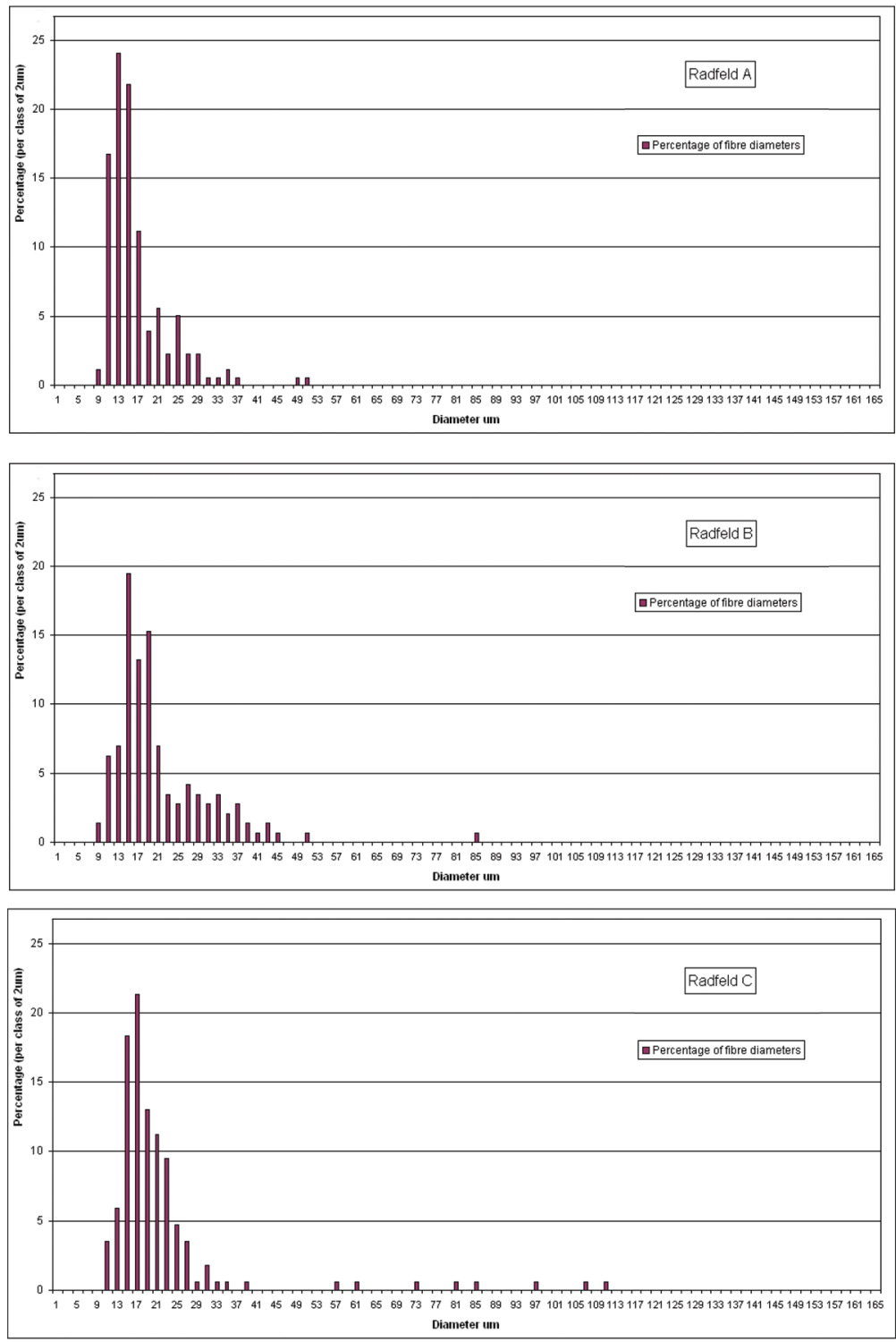

Abb. 9: Wollfeinheitsmessungen: Histogramme der Gewebe A, B und C (Grafik: M. Gleba-Univ. Cambridge)

\section{Nähnadel aus Bronze mit Fadenrest}

Im Zusammenhang mit der Verarbeitung von Textilien oder auch Leder ist eine Nähnadel aus Bronze zu sehen (Abb. 11), die bei einer Grabungskampagne 2008 in der Schlackensandhalde aufgefunden wurde (Schnitt 10, Abb. 2). Bemerkenswert sind die Überreste eines Fadens, der noch im Nadelöhr erkennbar ist ${ }^{28}$, und dessen Erhaltung auf die konservierende Wirkung der Kupfersalze zurückzuführen ist.

28 Fadenreste konnten bislang nur zur Sicherung von bronzenen Schmucknadeln beobachtet werden, siehe Ruckdeschel 2010.
Der feine Faden war dreimal kreuzweise im Bereich des Öhres um die Nadel gewickelt. Die Untersuchung im Rasterelektronenmikroskop (Abb. 12) ergab als Fadenmaterial pflanzliche Fasern, eventuell Brennnesselfasern. Es handelt sich um feines, 0,3 mm dünnes Garn, das in s-Drehung gesponnen wurde. Offensichtlich wurde mit der Nadel mit einem Faden aus Pflanzenfasern genäht, wobei sich pflanzliches Material durch seine Zugfestigkeit und Belastbarkeit sehr gut eignet. Nach Beendigung der Näharbeit wurde der Fadenrest zur Aufbewahrung um die Nadel gewickelt.

Die Bronzenadel lässt bei genauer Betrachtung Details ihrer Herstellung erkennen. So zeigt sie unter dem Mikroskop in Längsrichtung parallel verlaufende Rillen, die auf den Schleifvorgang bei der Herstellung hindeuten. Im 

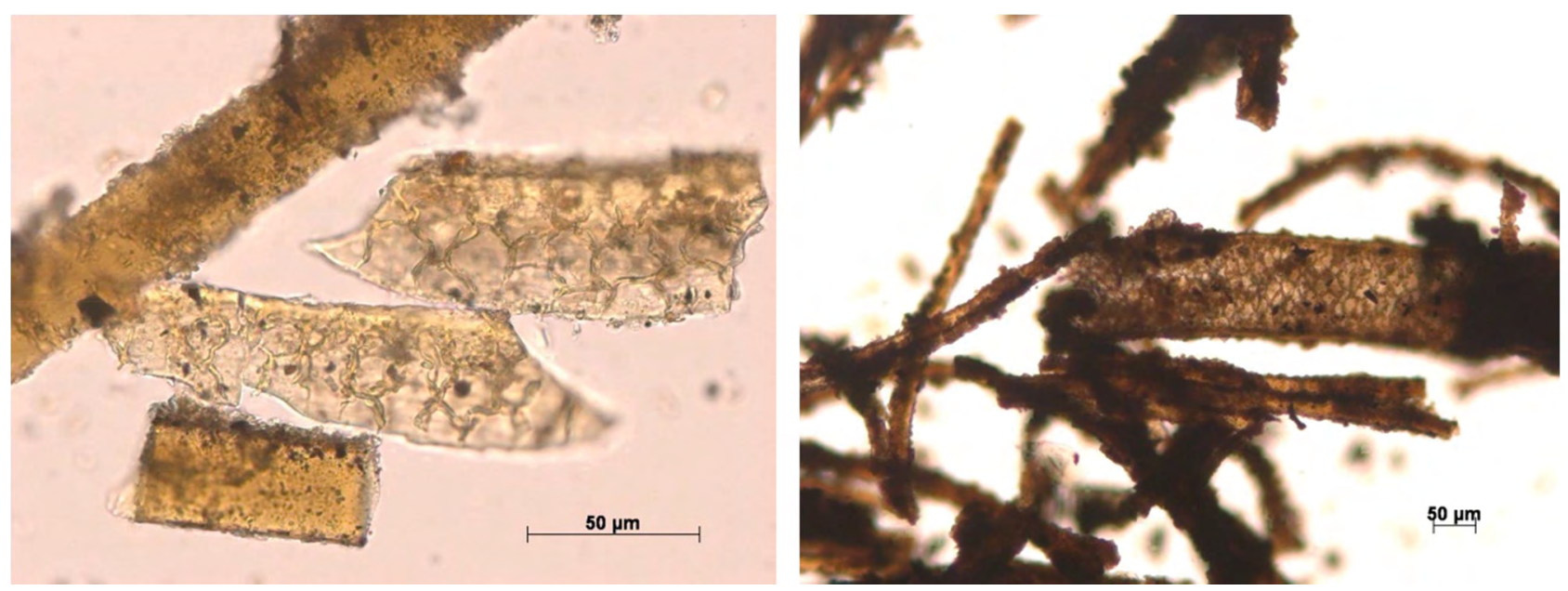

Abb. 10: Radfeld: Durchlichtmikroskop-Aufnahmen der Fasern der Gewebe A und B. Bruchstücke von stark abgebauten und kontaminierten Wollfasern. Rechts: Gewebe A, helle Fasern; links: Gewebe B, dunkelbraune Fasern (Durchlichtmikroskopische Aufnahme: R. Hofmann-de Keijzer-ARCH Vienna)

Bereich der Nadelspitze verlieren sich die Rillen, was sich durch das Zuschleifen der Spitze und/oder den Gebrauch erklären lässt. Die „Politur“ der Nadelspitze könnte auf eine Lederarbeit zurückzuführen sein, zu denken wäre beispielsweise an die Anfertigung und Reparatur von Blasebälgen aus Tierhäuten. Der Nadelkopf ist auf einer Länge von $2 \mathrm{~cm}$ mit rechteckigem Querschnitt ausgeschmiedet, das Öhr durch Spaltung mit schlitzförmiger Lochung hergestellt. Die 6,5 cm lange Bronzenadel kann sowohl zum Nähen von gröberen Textilien wie auch von Leder eingesetzt worden sein. Nach einer halbquantitativen Analyse besteht die Nadel aus Zinnbronze mit deutlichen Spuren von Blei ${ }^{29}$. Es ist deshalb anzunehmen, dass die Nadel nicht vor Ort aus dem lokal produzierten Kupfer hergestellt worden ist, sondern aus einer handelsüblichen Zinnbronze, und wahrscheinlich als Fertigprodukt auf den Verhüttungsplatz gelangt ist. Nadeln mit Öhr sind im Nordtiroler Raum beispielsweise aus urnenfelderzeitlichen Grabkomplexen bekannt ${ }^{30}$ und finden dann vor allem in der Latènezeit (meist aus Eisen) Verbreitung ${ }^{31}$.

29 Rasterelektronenmikroskop, Mathias Mehofer VIAS - Vienna Institute for Archaeological Science.

30 Z. B. Volders, Grab 84: Kasseroler 1959, 224 Taf. IV,84; Sperber 1977, 422.

31 Z.B. Kundl, Grab 132: Lang 1998, 144 Nr. 1345/4. - Siehe auch Töchterle 2009, 102.

\section{Vergleiche: Textilien als Dichtungsmaterial, Recycling von Textilien}

Gerade Wollgewebe eignen sich als Dichtungsmaterial besonders, da sie eine große Flexibilität aufweisen, bei entsprechender Verdrehung aber auch gut abdichten. Gewebe in ähnlicher Funktion wurden auch beim bronzezeitlichen Kupferbergbau am Mitterberg ${ }^{32}$ gefunden. In diesem Falle befand sich der Textilrest untertage im Berg und nicht bei einer obertägigen Aufbereitungsanlage. Das Mitterberger Gewebe war zwischen den Planken einer Verdämmung entdeckt worden, einer Vorrichtung im Bergbau gegen das Eindringen von Tagwasser. Dort diente der Textilrest offensichtlich gemeinsam mit Moos als Dichtungsmaterial in den lehmverschmierten Fugen der Bretterwand.

Eine Verwendung von Textilien als Abdichtung ist auch im antiken und historischen Bootsbau bekannt, was ebenfalls auf die gute abdichtende Wirkung verweist. Bei der Auffindung des Nydam-Bootes in einem Moor in Dänemark, datiert zwischen 310 und $320 \mathrm{n}$. Chr. ${ }^{33}$, wurden zwischen den Planken verschiedene Textilien entdeckt, die dort zur Abdichtung hineingestopft worden waren. Es handelt sich um keinen bestimmten Gewebetyp, auch Abriebspuren an den Fetzen zeigten deutlich, dass hier recyceltes Material benützt wurde.

32 Klose 1916, 16: Befund des Dammes und 35: Beschreibung des Textilrestes.

33 Möller-Wiering 2011, 93-94. 
A

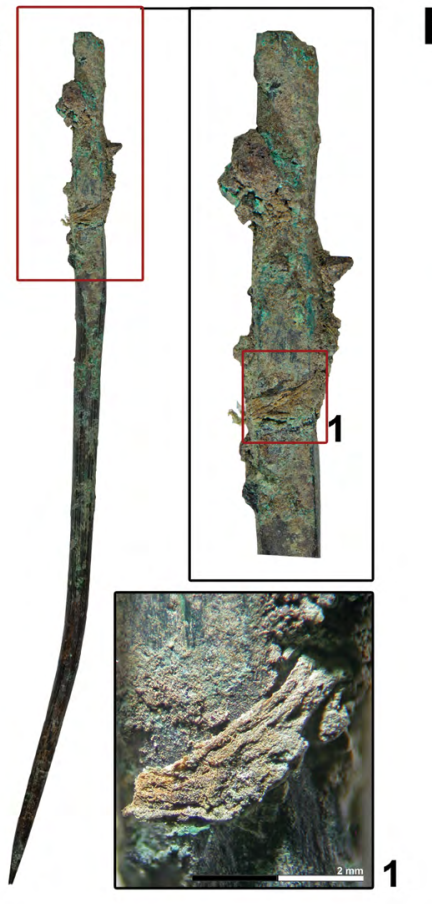

(1)
B

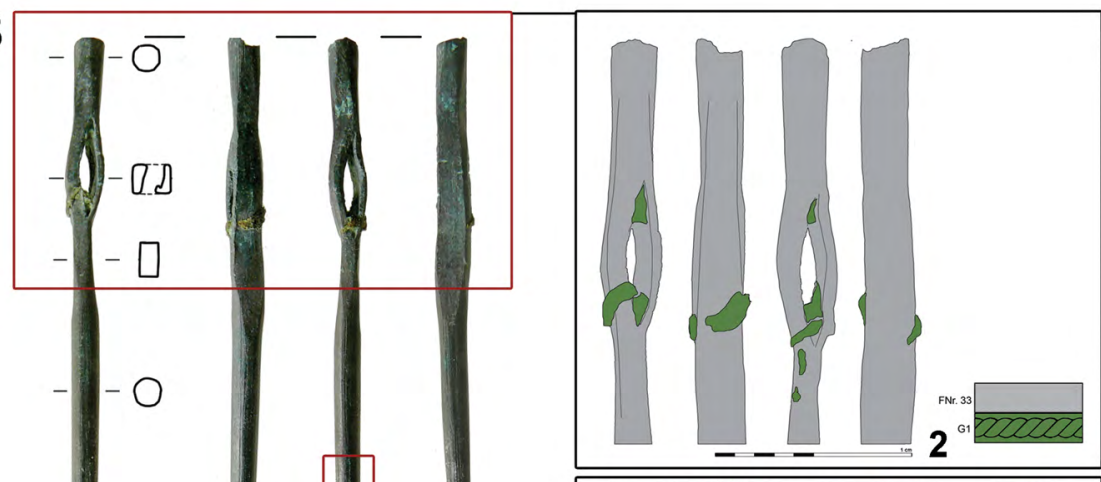

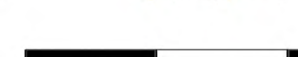

Abb. 11: Nähnadel aus Bronze mit Öhr und Fadenrest vor (A) und nach (B) der Restaurierung; 1. Detail der teilweise mineralisierten Faserstruktur; 2. Kartierung der erhaltenen organischen Faserauflagen; 3. Schleifspuren vom Herstellungsprozess; 4. -5. Gebrauchsspuren an der Nadelspitze (Fotos: G. Goldenberg, U. Töchterle-Univ. Innsbruck)
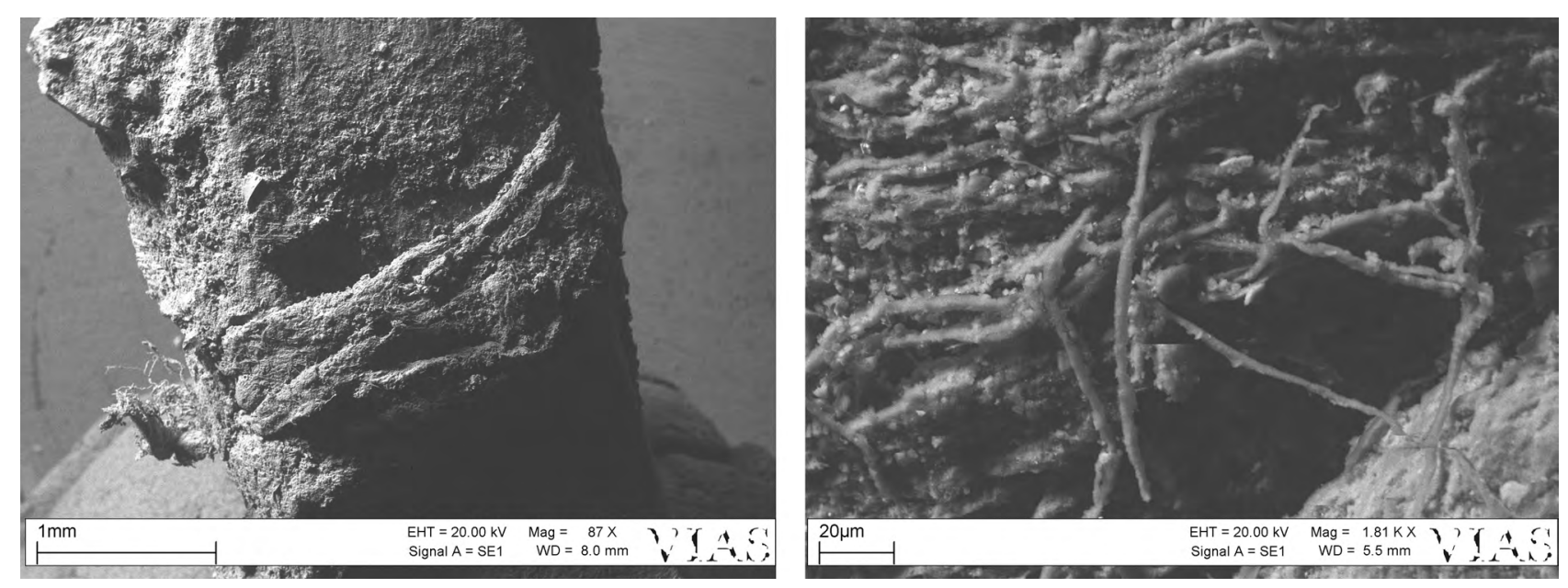

Abb. 12: Nadel und Detailbild der Fadenwicklung (Fotos: M. Mehofer-VIAS Wien)

Sehr wahrscheinlich stellt diese letztendliche Verwendung der Textilien von Radfeld Mauk A als Dichtung nicht den primären Zweck der Gewebe dar. Gezieltes „Recycling“von Stoffen kennen wir etwa aus den prähistorischen Salzbergwerken Hallstatt ${ }^{34}$ (Abb. 13) und Dürrnberg ${ }^{35}$. Es

34 Reschreiter/Grömer/Totschnig 2009, 312-314. 35 Stöllner 2005, Abb. 12. wurden im Abraum der Bergbaue Gewebe gefunden, die offensichtlich zu einem Gutteil bereits als Lumpen zu verschiedenen, heute nur noch schwer nachzuvollziehenden Zwecken in den Berg gelangten. Manche Stoffe wurden auch in Streifen gerissen und teilweise verknotet, um etwa als Bindematerial zu dienen. Ein Gewebe vom Dürrnberg wurde in seiner Sekundärfunktion als Wundverband für einen Finger verwendet. 

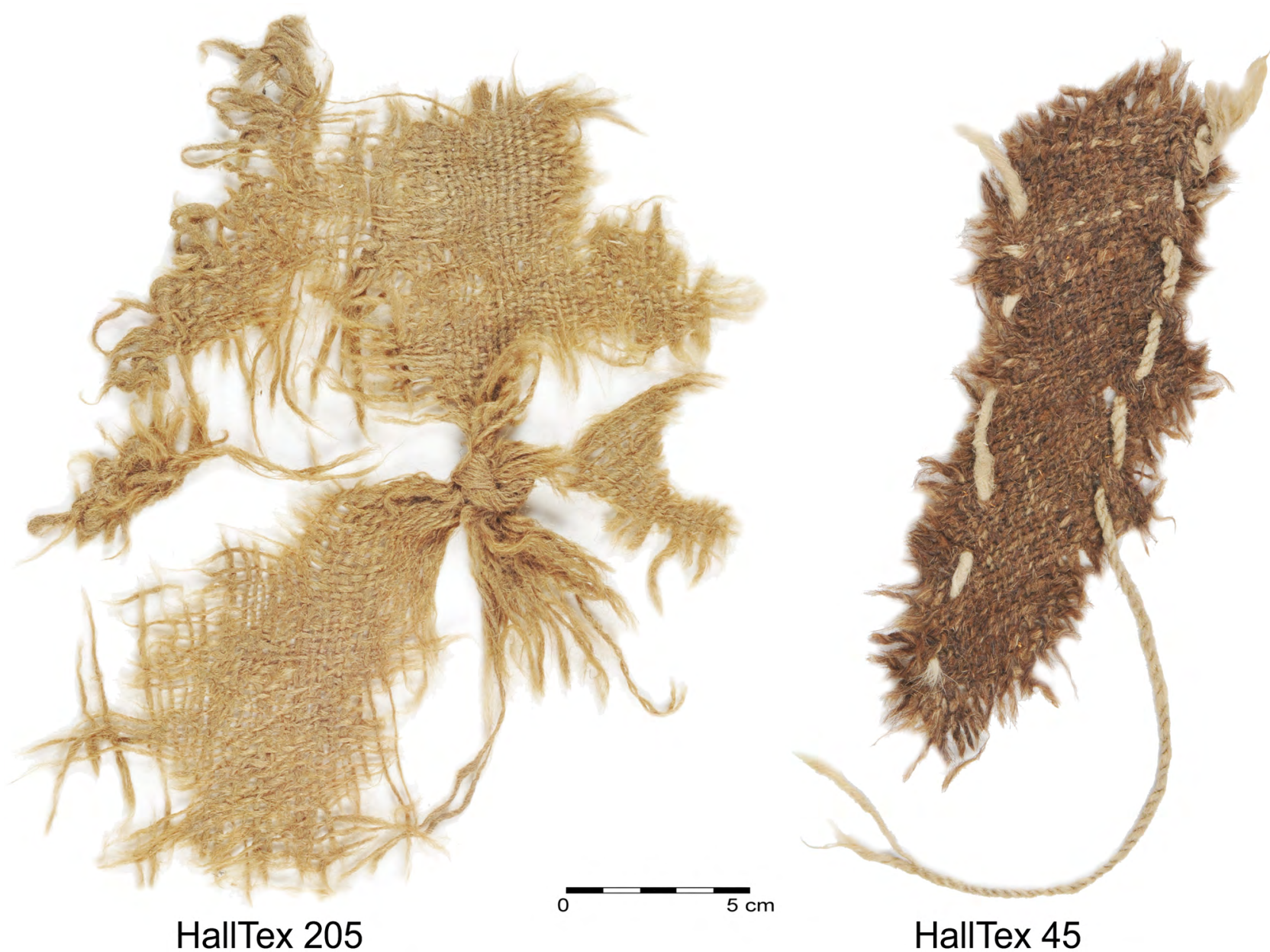

Abb. 13: Hallstatt, bronzezeitlicher Salzbergbau: „Recycelte“ Textilien: HallTex 45 mit sekundärer Naht, HallTex 205 mit Knoten (Fotos: A. Rausch-NHM Wien)

Bei den Funden aus Radfeld Mauk A gibt es verschiedene Interpretationsmöglichkeiten, welche Funktion die Gewebe vor ihrer endgültigen Verwendung als Abdichtung hatten. Die technischen Details der Stoffe sprechen dafür, dass es sich eventuell um Fetzen abgetragener Kleidung handelt, da derlei Qualitäten bei den original erhaltenen Kleidungsstücken aus der Bronzezeit durchaus geläufig sind. Einschränkend muss betont werden, dass die vollständigen Gewänder aus den Baumsärgen Dänemarks ${ }^{36}$ um 1300-1100 v. Chr. kulturell eher weit entfernt sind. Durch die Sitte der Brandbestattung in der mitteleuropäischen Urnenfelderzeit wissen wir meist nicht, welche Stoffe in unserem Raum konkret als Kleidung verwendet wurden. Lediglich ein neuerer Fund aus Grundfeld in Deutschland ${ }^{37}$ kann hier herangezogen werden. In einer reich mit Bronzeschmuck und Trachtbestandteilen aus- gestatteten frühurnenfelderzeitlichen Körperbestattung wurden Kleidungsreste in Form von einfachem Leinen entdeckt, auch die Reste des Gürtels konnten als Textilband identifiziert werden. Es handelt sich also auch hier, bei einem reicheren Frauengrab, um leinwandbindige Kleidungsstoffe, vergleichbar mit den verschiedenen Qualitäten aus Radfeld Mauk A.

Andererseits könnte es sich bei den Geweben in ihrer Erstverwendung auch um spezialisiertes Gerät aus dem Bergbaubetrieb gehandelt haben. $\mathrm{Zu}$ denken wäre an Tücher, wie sie vom Goldwaschen auf „Waschherden“ etwa auch von mittelalterlichen/frühneuzeitlichen Befunden bekannt sind ${ }^{38}$ (Abb. 14). 


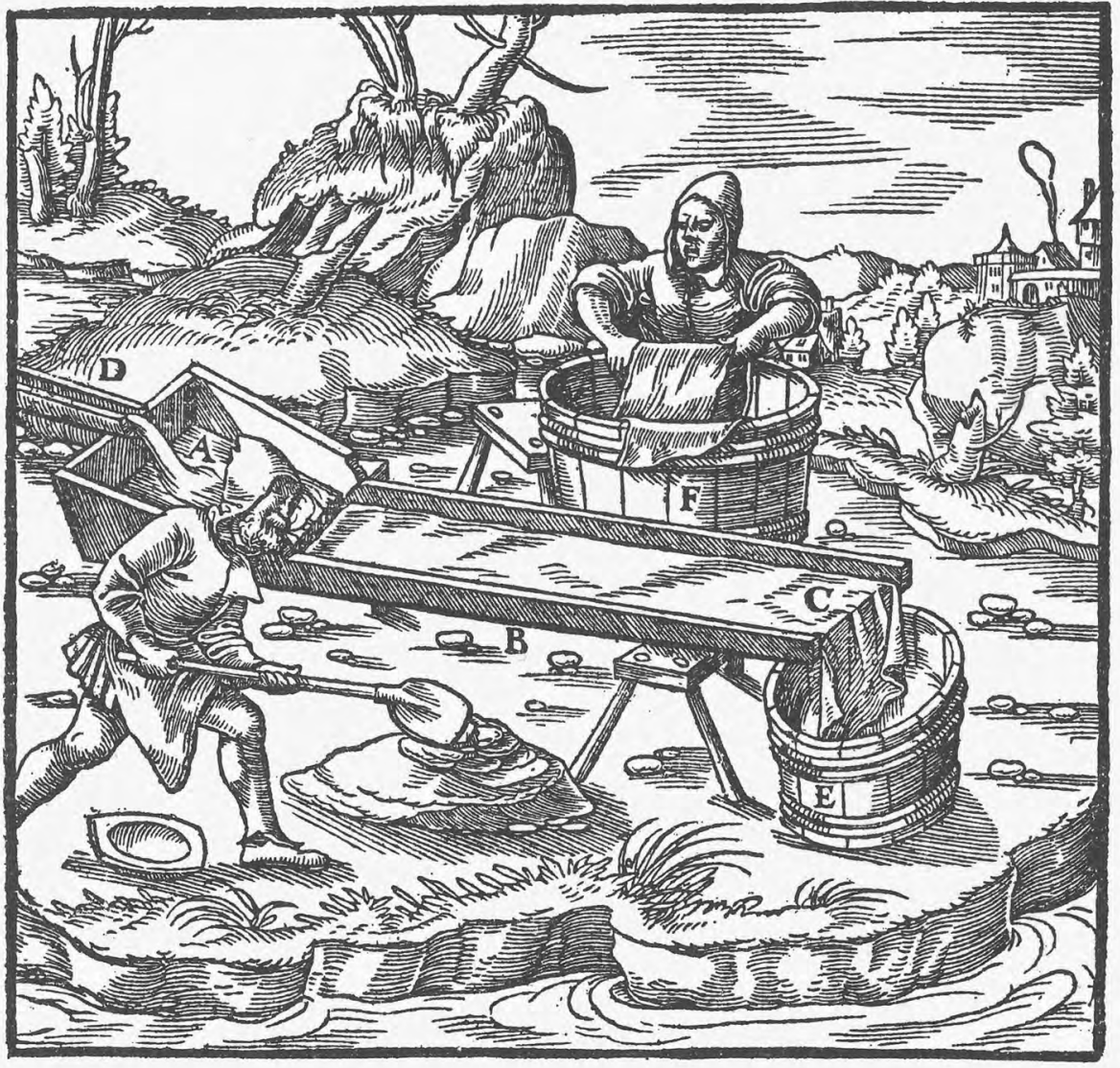

Das Haupt des Herdes A. Der Herd B. Das Tuch C. Das kleine Gerinne D. Das unter dem Herde aufgeftellte $F a \beta E . \quad$ Das Faß, in dem des Tuch gewaschen wird F.

Abb. 14: Goldwaschen in einer Holzrinne (Herd B) auf einem Tuch (C) nach Agricola 1556

\section{Die Textilfunde von Radfeld Mauk A im Kontext bronzezeitlicher Textilfunde in Europa}

Während der nordeuropäische Raum ${ }^{39}$, besonders Dänemark und Schleswig-Holstein, eine starke Forschungstradition in Bezug auf bronzezeitliche Textilien aufweist - es sind relativ viele Textilfunde, darunter vollständig erhaltene Kleidungsstücke aus den Baumsärgen bekannt - sind aus Mittel- und Südeuropa vergleichsweise nur wenige Funde publiziert. Erst in den letzten Jahren wurden einige bedeutende Fundkomplexe vorgelegt, wie die Textilien aus den frühbronzezeitlichen Pfahlbausiedlungen Nord-

39 Baumsargfunde: Hald 1980; Mannering et al. 2012. - Sonstige Funde aus Skandinavien: Bender Jørgensen 1986, 185-194; 298-301. italiens ${ }^{40}$ oder von den mittelbronzezeitlichen Bereichen des Salzbergwerkes in Hallstatt ${ }^{41}$.

Allgemein passen sich die Funde aus Radfeld in Fadenstärke als auch Gewebedichte gut in die Textilqualitäten der Bronzezeit Mitteleuropas ein (Abb. 15), wobei als geografisch nächste Vergleiche Hallstatt und das Kupferbergwerk am Mitterberg ${ }^{42}$ herangezogen werden können. Auch die Faserqualitäten mit der Mischung von feinen und groben Fasern sind von den genannten Fundorten gut bekannt ${ }^{43}$. Ebenso ist für die Bronzezeit Leinwandbindung charakteristisch.

Der bisherige Forschungsstand geht aufgrund der bronzezeitlichen Textilfunde von unterschiedlichen Webstuhltechnologien in Nordeuropa und Mitteleuropa aus. Aus Nordeuropa liegen ausschließlich Wollgewebe vor,

40 Bazzanella/Mayr 2009, 75-138, Katalog.

41 Grömer et al. 2013.

42 Dies. 2009, Abb. 6.

43 Grömer 2016, Abb. 26. 

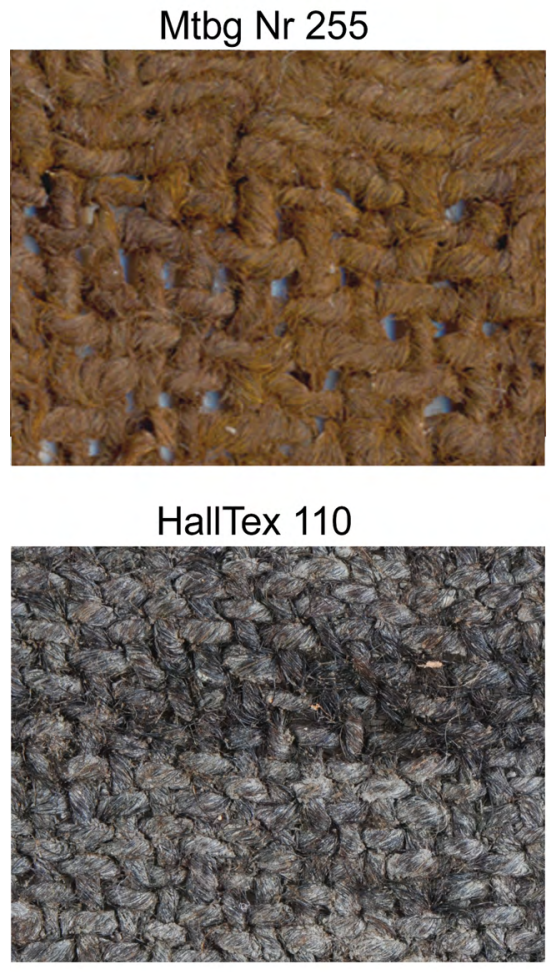

HallTex 45

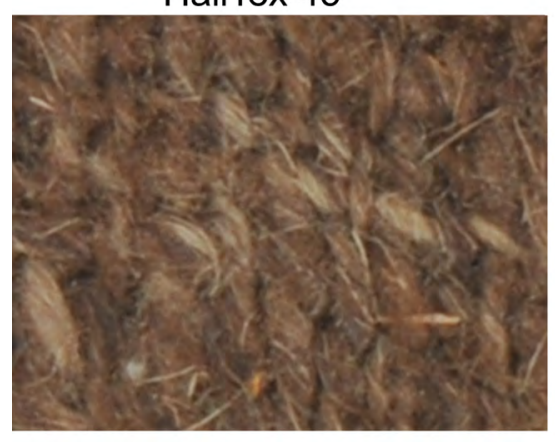

\section{HallTex 208}

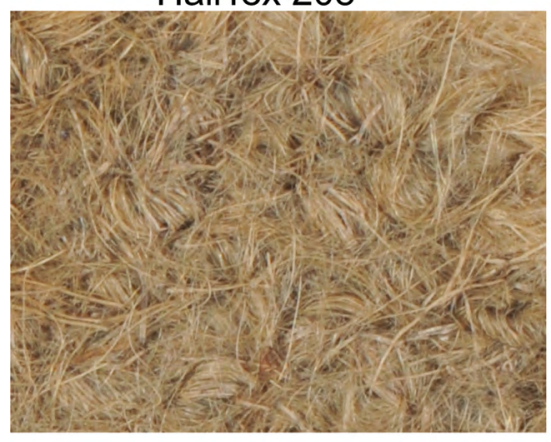

Mtbg Nr 105

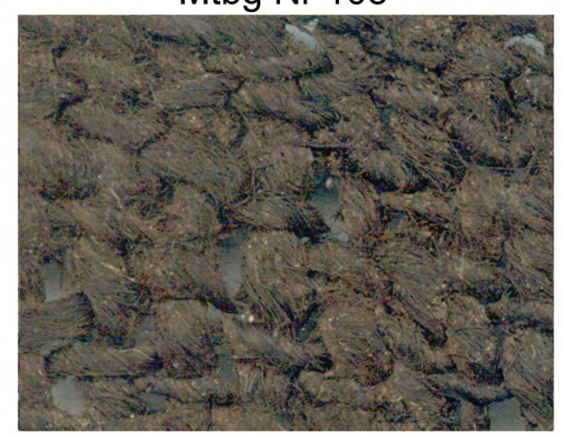

HallTex 224

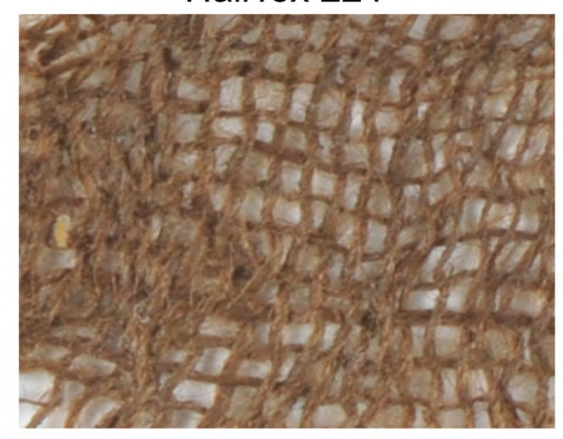

HallTex 205

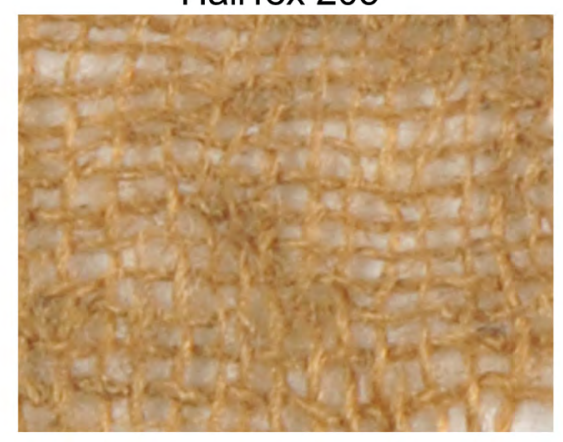

HallTex 211

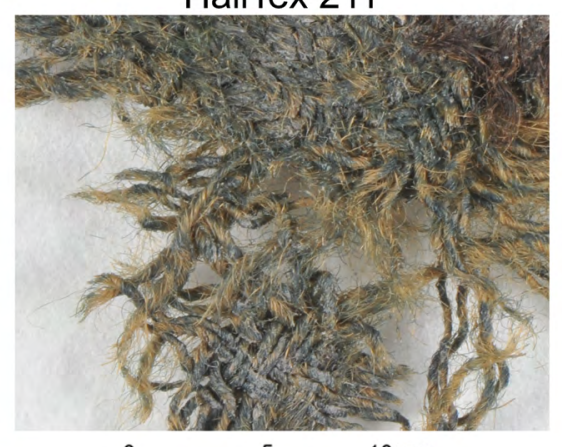

5

$10 \mathrm{~mm}$

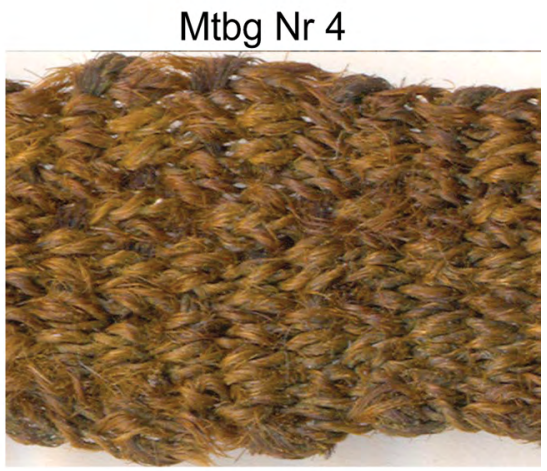

HallTex 201

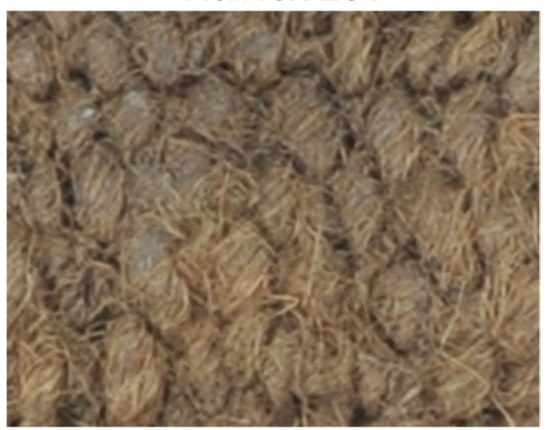

HallTex 22

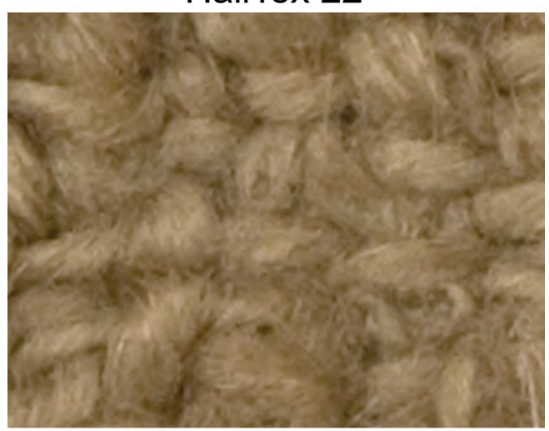

HallTex 27

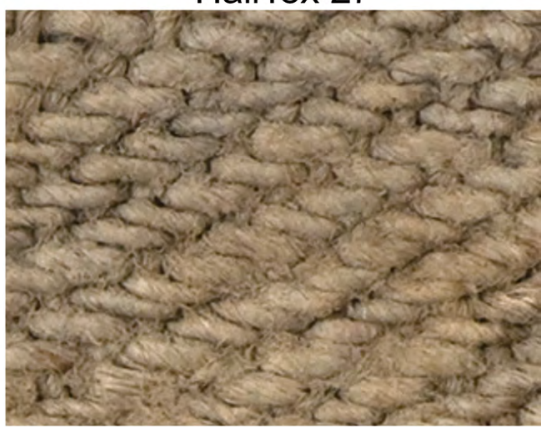

Abb. 15: Bronzezeitliche Textilien Mitterberg und Hallstatt (Fotos: A. Rausch-NHM Wien) 
für die in Kette und Eintrag einfaches Garn verwendet wurde. In Mitteleuropa lassen sich für das Neolithikum und auch für die Frühbronzezeit vorwiegend Leinengewebe mit Zwirn (zwei versponnene Garne) in Kette und Schuss nachweisen ${ }^{44}$. Während der Gewichtswebstuhl in Mitteleuropa seit dem Neolithikum zur Herstellung von Leinen- später auch Wollgeweben verwendet wurde ${ }^{45}$, geht man davon aus, dass die bronzezeitlichen Wollgewebe aus Nordeuropa auf dem Rundwebstuhl hergestellt wurden $^{46}$. Bisher ist nicht genau bekannt, ab wann genau in Mitteleuropa Wolle zur Herstellung von Geweben eingeführt wurde und welche webtechnologischen Veränderungen damit einhergingen. In diesem Zusammenhang werden immer wieder die frühesten Wollfunde, besonders der neolithische Fund aus Wiepenkathen in Deutschland ${ }^{47}$ diskutiert. Von den österreichischen Kupferbergbauen (Mitterberg, um 1600 v. Chr.), Radfeld Mauk A (11. Jh. v. Chr.) und den bronzezeitlichen Bereichen aus Hallstatt (1500-1200 v. Chr.) sind nun fast ausschließlich Wollgewebe bekannt. In Mitteleuropa sind schließlich Wollgewebe in der Hallstattzeit sehr charakteristisch, wenn dann aber in der Webtechnik die Köperbindung die Leinwandbindung als vorherrschende Webart ablöst.

Auch die bei den Wollfeinheitsanalysen festgestellten Ergebnisse reflektieren gut den bisherigen Forschungsstand. In Mitteleuropa kann allgemein ein Übergang von einem bronzezeitlichen Vliestyp mit extrem feiner Unterwolle und groben Grannenhaaren zu den eisenzeitlichen Vliesen mit etwas gröberer Unterwolle und dem weitgehenden Fehlen von Grannenhaaren festgestellt werden ${ }^{48}$. Die Übergangstypen finden sich in den Wollen von Radfeld Mauk A wieder. Ob dies rein als eine Entwicklung der Schafrassen zu interpretieren ist ${ }^{49}$ oder auch andere Mechanismen, wie das Sortieren und Aufbereiten der Wolle $^{50}$ eine Rolle spielen, wird durch weitere Funde zu klären sein. Ob die auf dem Verhüttungsplatz zahlreich gefundenen Tierknochen (Speisereste) mit einem Anteil von ca. 38 \% Ovicapriden bzw. Schaf/Ziege ${ }^{51}$ unter Umständen auch mit einer lokalen Wollproduktion in Verbindung gebracht werden können, war bislang nicht Gegenstand der archäozoologischen Untersuchungen.

Die Funde aus Radfeld Mauk A waren auch möglicherweise mit gelben und/oder roten Farbstoffen gefärbt.

44 Bazzanella/Mayr 2009, 75-138; Rast-Eicher 2005, 117-120. 45 Vgl. Webgewichtfunde in Grömer 2016, 110-112.

46 Stærmose-Nielsen 1999, 124-125.

47 Bender Jørgensen/Rast-Eicher 2015, 67-68.

48 Vgl. Rast-Eicher 2008; 2013

49 Ryder 1964; 1983.

50 Rast-Eicher 2013, 168-172.

51 Schibler et al. 2011.
Auch dies ist bereits aus anderen bronzezeitlichen Textilkomplexen in Mitteleuropa belegt ${ }^{52}$. Zumindest in Pustopolje, Bosnien-Herzegowina (17. Jh. v. Chr.) und am Mitterberg sind gelbe und rote Farbkomponenten identifiziert worden, in Hallstatt zusätzlich auch blau mit Waid sowie braun mit Tanninen. Gerade in Hallstatt, von wo wir an die 80 bronzezeitliche und 230 eisenzeitliche Textilien besitzen, haben wir einen guten Überblick, welcher Prozentsatz der Gewebe gefärbt war. Bei den farbstoffanalysierten bronzezeitlichen Stücken sind nur wenige mit Farbstoffen identifiziert worden, während hingegen ein Großteil der eisenzeitlichen Textilien gefärbt war. Man kann also sagen, dass in der Bronzezeit zwar die ersten Versuche zur Textilfärbung gemacht wurden, dies aber als konkrete Technik zur farblichen Akzentuierung von Stoffen erst in der Eisenzeit eine starke Rolle gespielt hat.

Die Gewebe aus Radfeld Mauk A sind hier nun ein wichtiger „missing link“ bei der Klärung der Fragen, wie sich die Textiltechnologie von der Bronze- zur Eisenzeit entwickelt hat. Die vornehmlich so unscheinbaren Textilreste von Radfeld Mauk A stellen damit einen sehr kleinen aber dennoch wichtigen Baustein in der Grundlagenforschung zu bronzezeitlichen Textilien in Mitteleuropa dar, da derartige Funde bislang sehr rar sind.

\section{Danksagung}

Für die finanzielle Förderung der montanarchäologischen Forschungen im Raum Radfeld/Brixlegg in Nordtirol ist dem österreichischen Wissenschaftsfonds FWF, dem Land Tirol (TWF) und der Universität Innsbruck zu danken. Unterstützt und gefördert wurden die archäologischen Grabungen von der Gemeinde Radfeld und der Agrargemeinschaft Lehensassen, Radfeld. An der Grabungsdokumentation unter der jeweiligen Leitung von Gert Goldenberg waren u.a. beteiligt: Brigitte Rieser (1995), Petra Fliri (1997) und das Team des SFB HiMAT, Gerhard Tomedi, Simon Hye, Michael Klaunzer, Ulrike Töchterle und Markus Staudt (2007-2011).

Die Wollfeinheitsanalysen waren Teil des Forschungsprojektes FIBRE (Textile Fibre in Italy before Roman Empire) von Margarita Gleba, unterstützt durch den European Commission Marie Curie Intra-European Scholarship am Institute of Archaeology, University College London (2009-2011).

Einige Analysen an den Radfeld-Textilien wurden 2011 durch Mitglieder des Projektes CinBA durchgeführt

52 Vgl. Hofmann-de Keijzer 2016, Abb. 84. 
(Sophie Bergerbrant, Karina Grömer, Sølvi Helene Fossøy). CinBA - Creativity and Craft Production in Middle and Late Bronze Age Europe, HERA-Projekt 2010-2013, Nr. 09-HERA-JRP-CI-FP-020. Projektleitung Joanna Sofaer, Universität Southampton.

\section{Katalog der Textilfunde der Grabung Mauk A}

Die erste Beschreibung der Textilien erfolgte im feuchten Zustand bei der Konservierung. Diese Daten sind im Großen und Ganzen hier wiedergegeben. Bei einer zweiten Analyse nach zehn Jahren wurden die Gewebe im stark getrockneten Zustand erneut beschrieben. In den Anmerkungen werden jene Daten angegeben, die sich verändert haben. Zusätzlich zu den zuerst erhobenen Daten wurden noch der Drehwinkel und die Einzelgrößen der Stücke ausgemessen.

\section{Gewebe A}

Textilbeschreibung: mehrere größere und kleinere Fragmente eines groben Textiles in flächiger Leinwandbindung, teils noch stark zusammengefaltet und mit Lehm versetzt

Farbe und Material: hellbraun (feuchter Zustand), Wolle, feine Fasern

Größe (größere Teile): 6,2×2,5 cm; 2,8×2,2 cm

Anzahl Einzelteile: 7

Identifizierung Kette und Schuss: nein

Gewebetechnische Details:

\begin{tabular}{lll}
\hline & Fadensystem 1 & Fadensystem 2 \\
\hline Garn/Zwirn & $\mathrm{G}$ & $\mathrm{G}$ \\
Fadendrehung & $\mathrm{Z}$ & $\mathrm{S}$ \\
Drehwinkel & $25-33^{\circ}$ & $28-33^{\circ}$ \\
Fadenstärke & $0,8-0,9$ & $0,7-0,9$ \\
Gewebedichte & $9-10$ & Nicht auszählbar \\
(Fäden pro cm) & & \\
\hline
\end{tabular}

Bindungsart: Leinwandbindung

Oberflächenbesonderheiten: stark aufgelöst. Eventuell ist eine Webkante am größeren Stück erhalten

Anmerkungen: das Gewebe hat im getrockneten Zustand ein schwarzbraunes Aussehen; Fadenstärke am trockenen Gewebe: 0,6-0,9 mm

\section{Gewebe B}

Textilbeschreibung: mehrere größere und kleinere Fragmente eines mittelfeinen Textiles in Leinwandbindung, sehr dichtes Gewebe, stark verfaltet

Farbe und Material: hellbraun, Wolle; Mischfasern: hellbraune lange feine Fasern und gröbere hellbraune Elemente in Kette; hellbraune feine Fasern und kräftige schwarzbraune Fasern mit rundem Durchmesser in Schuss

Größe (größere Teile): 10,5×5 cm; 4×1,2 cm

Anzahl Einzelteile: 2 größere, 4 kleine Fragmente

Identifizierung Kette und Schuss: ja (aufgrund des Webfehlers)

Gewebetechnische Details:

\begin{tabular}{lll}
\hline & Kette & Schuss \\
\hline Garn/Zwirn & $\mathrm{G}$ & $\mathrm{G}$ \\
Fadendrehung & $\mathrm{z}$ & $\mathrm{z}$ \\
Drehwinkel & $35-40^{\circ}$ & $27-48^{\circ}$ \\
Fadenstärke & $0,5-0,8$ & $0,7-0,9$ \\
& $(\mathrm{z} . \mathrm{T} .0,5 \mathrm{~mm})$ & $($ z. T. 0,5-1 mm) \\
Gewebedichte & 11 & $8-12$ \\
(Fäden pro cm) & & (durchschnittlich 10) \\
\hline
\end{tabular}

Bindungsart: Leinwandbindung

Oberflächenbesonderheiten: Im Verlauf eines Fadensystems verschiedene Webfehler: doppelt eingezogene Garne; Garn springt teils über mehrere Eintragsfäden (3-6)

Anmerkungen: das Gewebe hat im getrockneten Zustand ein dunkelbraunes Aussehen; als Gewebedichte konnten nun je 10 Fäden pro cm ausgezählt werden

\section{Gewebe C}

Textilbeschreibung: viele kleine Fragmente eines sehr groben leinwandbindigen Gewebes mit sehr ungleichmäBiger Garnstärke

Farbe und Material: Wolle, Mischfasern: hellbraune feine Fasern und hellbraune gröbere Elemente Größe (größere Teile): 5×3,5 cm; 4,5×0,7 cm; $7 \times 1 \mathrm{~cm} ; 5 \times 2,5$ $\mathrm{cm} ; 4,5 \times 2 \mathrm{~cm}$

Anzahl Einzelteile: 21

Identifizierung Kette und Schuss: nein

Gewebetechnische Details:

\begin{tabular}{lll}
\hline & Fadensystem 1 & Fadensystem 2 \\
\hline Garn/Zwirn & $\mathrm{G}$ & $\mathrm{G}$ \\
Fadendrehung & $\mathrm{S}$ & $\mathrm{S}$ \\
Drehwinkel & $32-38^{\circ}$ & $35-40^{\circ}$ \\
Fadenstärke & $1,1-2$ & $1-2($ meist $1,5 \mathrm{~mm})$ \\
Gewebedichte (Fäden & $5-6$ & $4-5$ \\
pro $\mathrm{cm})$ & & \\
\hline
\end{tabular}


Bindungsart: Leinwandbindung

Oberflächenbesonderheiten: möglicherweise eine Webkante sichtbar

Anmerkungen: das Gewebe hat im getrockneten Zustand ein dunkel gelbbraunes bis schwärzliches Aussehen; Fadenstärke am trockenen Gewebe: 1,1-1,6 cm

\section{Bronzenadel Fnr. 33}

Faden am Hals einer Bronzenadel; feiner Faden dreimal rundumgewickelt

Farbe und Material: pflanzliche Faser (ev. Brennessel?)

Fadensystem 1: 0,3 mm s-Garn

Aufbewahrung: Institut für Archäologien, Univ. Innsbruck

\section{Literatur}

Agricola 1556: G. Agricola, De Re Metallica Libri XII - Zwölf Bücher vom Berg- und Hüttenwesen (1556). Übersetzt und bearbeitet von Carl Schiffner (Düsseldorf 1978).

Banck-Burgess 2000: J. Banck-Burgess, Bronzezeitliche TextilfundeRadfeld/Mauken. Unpubl. Arbeitsbericht (Esslingen 2000).

Bartel/Voß 2005: A. Bartel/H.Voß, Modisch in der Urnenfelderzeit: Zur Kleidung der Frau aus Grundfeld. Arch. Jahr Bayern 2005, 2005, 44-46.

Bazzanella/Mayr 2009: M. Bazzanella/A. Mayr, I reperti tessili, le fusaiole e i pesi da telaio dalla palafitta di Molina di Ledro (Trento 2009).

Bender Jørgensen 1986: L. Bender Jørgensen, Forhistoriske Textiler i Skandinavien. Prehistoric Scandinavian Textiles. Nordishe Fortidsminder Ser. B, Bd. 9 (Kopenhagen 1986).

-/Rast-Eicher 2015: -/A. Rast-Eicher, Searching for the earliest wools in Europe. In: K. Grömer/F. Pritchard (eds.), NESAT XII. The North European Symposium of Archaeological Textiles, $21^{\text {st }}-24^{\text {th }}$ May 2014 in Hallstatt, Austria. Archaeolingua Main Ser. 33 (Budapest 2015).

Gleba 2012a: M. Gleba, Fibre Analysis on textiles from Radfeld (Austria). Unpubl. Bericht, (Cambridge 2012).

- 2012b:-, From textiles to sheep: investigating wool fibre development in pre-Roman Italy using scanning electron microscopy (SEM). Journal Arch. Scien. 39, 2012, 3643-3661.

Goldenberg 2008: G. Goldenberg, Urgeschichtlicher Kupfererzbergbau bei Radfeld. In: Dorfbuch Radfeld (Radfeld 2008) 65-70.

- 2013: -, Prähistorischer Fahlerzbergbau im Unterinntal Montanarchäologische Befunde. In: K. Oeggl/V. Schaffer, Montanwerke Brixlegg AG (Hrsg.), Cuprum Tyrolense. 5550 Jahre Bergbau und Kupferverhüttung in Tirol (Innsbruck 2013) 89-122.

- 2015: -, Prähistorische Kupfergewinnung aus Fahlerzen der Lagerstätte Schwaz-Brixlegg im Unterinntal, Nordtirol. In: T. Stöllner/K. Oeggl (Hrsg.), Bergauf Bergab - 10.000 Jahre Bergbau in den Ostalpen. Veröff. Dt. Bergbau-Mus. Bochum 207 (Bochum 2015) 151-163.
- et al. 2012: -/E. Breitenlechner/S. Deschler-Erb/K. Hanke/ G. Hiebel/H. Hüster-Plogmann/S. Hye/M. Klaunzer/K. Kovács/ M. Krismer/J. Lutz, A. Maass/M. Moser/K. Nicolussi/K. Oeggl/ E. Pernicka/Th. Pichler/N. Pöllath/J. Schibler/M. Staudt/ B. Stopp/A. Thurner/U. Töchterle/G. Tomedi/P. Tropper/ F. Vavtar/Th. Weinold, Prähistorischer Kupfererzbergbau im Maukental bei Radfeld/Brixlegg. In: G. Goldenberg/ U. Töchterle/K. Oeggl/A. Krenn-Leeb (Hrsg.), Forschungsprogramm HiMAT - Neues zur Bergbaugeschichte der Ostalpen. Arch. Österreichs Spezial 4 (Wien 2011) 61-110.

-/Rieser 2004: -/B. Rieser, Die Fahlerzlagerstätten von Schwaz/ Brixlegg (Nordtirol). Ein weiteres Zentrum urgeschichtlicher Kupferproduktion in den österreichischen Alpen. G. Weisgerber/G. Goldenberg (Hrsg.), Alpenkupfer - Rame delle Alpi. Anschnitt Beih. 17 (Bochum 2004). 37-52.

Grömer 2009: K. Grömer, Textilien der Bronzezeit in Mitteleuropa. Arch. Austriaca 90/2006 (Wien 2009) 31-72.

- 2014: -, Römische Textilien in Noricum und Westpannonien im Kontext der archäologischen Gewebefunde 2000 v. Chr. 500 n. Chr. in Österreich. Austria Ant. 5 (Graz 2014).

- 2016: -, The Art of Prehistoric Textile Making - The development of craft traditions and clothing in Central Europe. Veröff. Prähist. Abt. 5 (Wien 2016).

- et al. 2013: -/A. Kern/H. Reschreiter/H. Rösel-Mautendorfer (Hrsg.), Textiles from Hallstatt. Weaving Culture in Bronze and Iron Age Salt Mines. Textilien aus Hallstatt. Gewebte Kultur aus dem bronze- und eisenzeitlichen Salzbergwerk. Archaeolingua 29 (Budapest 2013).

Hald 1980: M. Hald, Ancient Danish Textiles from Bogs and Burials. Nat. Mus. Denmark XI, 1980.

Heiss/Oeggl 2005: A. Heiss/K.Oeggl, The oldest evidence of Nigella damascene L. (Ranunculaceae) and its possible introduction to central Europe. Vegetation Hist. and Archaeobot. 14, 2005, 562-570.

Hofmann-de Keijzer 2016: R. Hofmann-de Keijzer, Dyeing. In: K. Grömer (Hrsg.), The Art of Prehistoric Textile Making - The development of craft traditions and clothing in Central Europe. Veröff. Prähist. Abt. 5 (2016 Wien) 140-169.

- et al. 2012: -/M. van Bommel/I. Joosten, Farbstoffanalysen an den Textilien aus Radfeld. Unpubl. Ber. (Wien 2012).

Hye et al. 2009: S. Hye/G. Goldenberg/M. Klaunzer/U. Töchterle/ G. Tomedi, Montanarchäologische Untersuchungen im Bergbaugebiet Mauken bei Radfeld, Nordtirol. Der spätbronzezeitliche Schmelzplatz Mauk A. In: K. Oeggl/M. Prast (Hrsg.), Die Geschichte des Bergbaus in Tirol und seinen angrenzenden Gebieten. Proceedings zum 3. Milestone-Meeting des SFB HiMAT vom 23.-26. 10. 2008 in Silbertal (Innsbruck 2009) 311-316.

Kasseroler 1959: A. Kasseroler, Das Urnenfeld von Volders. Schlern-Schr. 204 (Innsbruck 1959).

Klose 1916: O. Klose, Die prähistorischen Funde vom Mitterberge bei Bischofshofen im städtischen Museum Carolino-Augusteum zu Salzburg und zwei prähistorische Schmelzöfen auf dem Mitterberge. In: G. Kyrle (Hrsg.), Österreichische Kunsttopographie XVII. Urgeschichte des Kronlandes Salzburg (Wien 1916) $1-40$.

Krismer et al. 2015: M. Krismer/G. Goldenberg/P. Tropper, Mineralogical-petrological investigations of metallurgical slags from the Late Bronze Age fahlore-smelting site Mauken (Tyrol, Austria). Anschnitt, Beih. 26, 2015, 309-318. 
Lang 1998: A. Lang, Das Gräberfeld von Kundl im Tiroler Inntal. Studien zur vorrömischen Eisenzeit in den zentralen Alpen. Frühgesch. u. Provinzialröm. Arch. Mat. u. Forsch. 2 (Rahden 1998).

Mannering et al. 2012: U. Mannering/M. Gleba/M. Bloch Hansen, Denmark. In: M. Gleba/U. Mannering (eds.), Textiles and Textile Production in Europe from Prehistory to AD 400. Ancient Textiles Ser. Vol. 11 (Oxford 2012) 91-121.

Möller-Wiering 2011: S. Möller-Wiering, War and Worship: Textiles from the $3^{\text {rd }}-4^{\text {th }}$ Century AD Weapon Deposits in Denmark and Northern Germany, Ancient Textiles Ser. 9 (Oxford 2011).

Mutschlechner 1984: G. Mutschlechner, Erzbergbau und Bergwesen im Berggericht Rattenberg. (Alpbach, Brixlegg, Rattenberg, Reith i. A. 1984).

Nicolussi/Pichler 2013: K. Nicolussi/Th. Pichler, Bergbauholz aus historischer und prähistorischer Zeit - Jahrringanalysen zur Bergbaugeschichte im Raum Schwaz-Brixlegg. In: K. Oeggl/ V. Schaffer, Montanwerke Brixlegg AG (Hrsg.), Cuprum Tyrolense. 5550 Jahre Bergbau und Kupferverhüttung in Tirol (Innsbruck 2013) 153-187.

-/Pichler/Thurner 2015:-/-/A. Thurner, Dendro-Daten zum prähistorischen Kupferbergbau in Westösterreich. In: T. Stöllner/ K. Oeggl (Hrsg.), Bergauf Bergab - 10.000 Jahre Bergbau in den Ostalpen. Veröff. Dt. Bergbau-Mus. Bochum 207 (Bochum 2015) 239-246.

Rast-Eicher 2005: A. Rast-Eicher, Bast before wool: the first Textiles. In: P. Bichler/K. Grömer/R. Hofmann-de Keijzer/A. Kern/H. Reschreiter (Hrsg.), “Hallstatt Textiles” Technical Analysis, Scientific Investigation and Experiment on Iron Age Textiles. BAR Int. Ser. 1351, 2005, 117-131.

- 2008: -, Textilien, Wolle, Schafe der Eisenzeit in der Schweiz. Antiqua 44 (Basel 2008).

- 2013: -, Die Faserqualität von Fellen und Textilien aus dem Salzbergwerk von Hallstatt. The fibre quality of skins and textiles from the Hallstatt salt mines. In: K. Grömer/A. Kern/ H. Reschreiter/H. Rösel-Mautendorfer (Hrsg.), Textiles from Hallstatt. Weaving Culture in Bronze and Iron Age Salt Mines. Archaeolingua 29, (Budapest 2013) 135-162.

Reschreiter/Grömer/Totschnig 2009: H. Reschreiter/K. Grömer/ R. Totschnig, Reich im Grab - Sparsam in der Grube. Überlegungen zum Ressourcenmanagement im ältereisenzeitlichen Salzbergwerk Hallstatt. In: R. Karl/J. Leskovar (Hrsg.), Interpretierte Eisenzeiten. Fallstudien, Methoden, Theorie. Stud. Kulturgesch. Oberösterr. 22, 2009, 307-320.

Retournard 2015: E. Retournard, Archaeological Textiles from an Ore-Washing Workshop in the French Alps. Arch. Textiles Rev. $57,2015,75-88$.
Ruckdeschel 2010: W. Ruckdeschel, Mit Nadel und Faden. Bayer. Vorgeschbl. 75, 2010, 9-40.

Ryder 1964: M. L. Ryder, Fleece evolution in domestic sheep. Nature 204/4958, 1964, 555-559.

- 1983:-, Sheep and Man (London 1983).

Schatz et al. 2002: I. Schatz/H. Schatz/F. Glaser/A. Heiss, Subfossile Arthropodenfunde in einer bronzezeitlichen Grabungsstätte bei Radfeld (Tirol, Österreich) - (Arcari: Oribatida; Insecta: Coleoptera, Hymenoptera: Formicidae). Ber. nat.-med. Verein Innsbruck 89, 2002, 249-264.

Schibler et al. 2011: J. Schibler/E. Breitenlechner/S. Deschler-Erb/ G. Goldenberg/K. Hanke/G. Hiebel/H. Hüster-Plogmann/ K. Nicolussi/E. Marti-Grädel/Th. Pichler/A. Schmidl/ S. A. Schwarz/B. Stopp/K. Oeggl, Miners and mining in the Late Bronze Age: a multidisciplinary study from Austria. Antiquity 85, 2011, 1259-1278.

Sperber 1977: L. Sperber, Nordtiroler Urnenfelderkultur. Unpubl. Manuskr:. Univ. Innsbruck (Innsbruck 1977).

Stærmose-Nielsen 1999: K.-H. Stærmose Nielsen, Kirkes væv. Opstadvævens historie og nutidige brug. (Engl. Begleitheft: The loom of circe. The history of the warp-weighted loom and its present use). Forsøg med fortiden 6, Historisk-Arkæologisk Forsøgscenter (Lejre 1999).

Stöllner 2005: Th. Stöllner, More than old rags - Textiles from the Iron Age Salt-mine at the Dürnberg (Mehr als alte Fetzen - Textilien aus dem eisenzeitlichen Salzbergwerk von HalleinDürrnberg. In: P. Bichler/K. Grömer/R. Hofmann-de Keijzer/ A. Kern/H. Reschreiter (Hrsg.), „Hallstatt Textiles“ Technical Analysis, Scientific Investigation and Experiment on Iron Age Textiles. BAR Int. Ser. 1351, 2005, 161-174.

Töchterle 2009: U. Töchterle, Kontinuität und Wandel. Funde aus prähistorischer Zeit. In: D. Mair/U. Töchterle, Archäologische Topographie der Siedlungskammer Ampass. Ikarus 4 (Innsbruck 2009).

- et al. 2013:-/G. Goldenberg/Ph. Schneider/P. Tropper, Spätbronzezeitliche Verhüttungsdüsen aus dem Bergbaurevier Mauken im Unterinntal, Nordtirol: Typologie, mineralogischpetrographische Zusammensetzung und experimentelle Rekonstruktionsversuche. Anschnitt 65/1, 2013, 2-19.

Walton Rogers 1998: P. Walton Rogers, Dyes and Wools in Three Bronze Age Textiles from Radfeld/Mauken, North-Tirol, Austria. Unpubl. Arbeitsbericht 1998. In: Banck-Burgess 2000, 10.

Walton/Eastwood 1988: P. Walton/G. Eastwood, The Cataloguing of Archaeological Textiles. Inst. Arch. Publ. (London 1988).

Weisgerber/Goldenberg 2004: G. Weisgerber/G. Goldenberg (Hrsg.), Alpenkupfer - Rame delle Alpi. Anschnitt Beih. 17 (Bochum 2004). 